

\section{ON THE DETERMINATION OF THE LONG PERIOD TIDAL PERTURBATIONS IN THE \\ ELEMENTS OF ARTIFICIAL EARTH SATELLITES}

\section{Peter Musen \\ and}

Theodore Felsentreger

May 1972

Goddard Space Flight Center Greenbelt, Maryland 


\section{NOTATIONS}

A - the right ascension of the satellite,

$r$ - the distance of the satellite from the center of the Earth,

$\mathbf{r}^{0}-$ the unit vector directed from the center of the Earth toward the satellite,

$\lambda, \mu, \nu$ - the equatorial components of $\mathbf{r}^{0}$,

$\mathrm{g}$ - the mean anomaly of the satellite,

$\mathrm{f}$ - the true anomaly of the satellite,

$\omega$ - the argument of perigee of the satellite,

- the right ascension of the ascending node of the satellite,

$\pi=\omega+\delta-$ the longitude of the perigee of the satellite,

$\mathrm{L}=\mathrm{g}+\pi-$ the mean longitude of the satellite,

$i$ - the inclination of the orbital plane of the satellite relative to the equatorial plane of a given epoch,

e - the eccentricity of the satellite's orbit,

a - the semi-major axis of the orbit of the satellite,

$\mathrm{n}$ - the mean motion of the satellite,

$\mathbf{R}$ - the unit vector normal to the orbital plane of the satellite,

$\delta \mathrm{L}, \delta \pi, \delta \delta, \delta \mathrm{i}$ - the tidal perturbations in $\mathrm{L}, \pi, \delta$ and $\mathrm{i}$, respectively, $m^{\prime}$ - the mass of the Moon (Sun),

$A^{\prime}$ - the right ascension of the moon (Sun),

$x^{\prime}, y^{\prime}, z^{\prime}-$ the equatorial rectangular coordinates of the Moon (Sun) referred to the mean equator and equinox of a given date, $r^{\prime}-$ the distance of the Moon (Sun) from the center of the Earth, 


$$
\begin{aligned}
& \lambda^{\prime}, \mu^{\prime}, \nu^{\prime} \text { - the equatorial components of the unit vector } \mathbf{r}^{\prime 0} \text { directed } \\
& \text { from the center of the Earth toward the Moon (Sun), } \\
& a^{\prime} \text { - the semi-major axis of the lunar (solar) orbit defined in such a } \\
& \text { manner that the constant part in the expansion of } a^{1} / r^{1} \text { be equal to one, } \\
& \text { G - the gravitational constant, } \\
& \text { M - the mass of the Earth, } \\
& \mathbf{R} \text { - the equatorial radius of the Earth, } \\
& \epsilon-\text { the eccentricity of the meridian of the Earth, } \\
& k_{2}, k_{3}, \cdots-\text { Love numbers, } \\
& a=\mathrm{R} / \mathrm{a} \text {, } \\
& \alpha^{\prime}=\mathrm{R} / \mathrm{a}^{\prime}-\text { the lunar (solar) parallactic factor, } \\
& \Omega \text { - the tidal disturbing function, } \\
& \frac{\partial \Omega}{\partial Z} \text { - the component of the disturbing force normal to orbital plane } \\
& \text { of the satellite; } \\
& \mathrm{P}_{\mathrm{nm}} \text { - associated Legendre functions, } \\
& \text { I - the idemfactor. }
\end{aligned}
$$




\section{INTRODUCTION}

In the present article we develop and extend the ideas on the tidal perturbations of artificial satellites formulated in a previous article by Musen and Estes (1971). The magnitude of the tidal effects depends upon the elastic properties of the Earth as described by Love numbers. The Love numbers appear as the coefficients in the expansion of the exterior tidal potential in terms of spherical harmonics (in Maxwellian form). In the previous article, we considered the Love numbers as constants over the whole Earth and substituted their "global" values into the potential and into the differential equations for the tidal perturbations in the motion of satellites.

These global values can be understood to be doubly averaged local Love numbers, the averaging process being performed over the whole surface of the Earth.

Such an approach yields many interesting and important results (Smith, Kolenkiewicz and Dunn, 1971). However, there are discrepancies between the values of Love numbers as obtained from the observations of satellites. This suggests a necessity for further sharpening the idea and making use of a single averaging process performed only along the parallels of latitude. In this respect, we follow in the footsteps of Kaula (1969).

This single averaging process preserves the additional long period tidal effects (with periods of a few days or more) which were omitted in the previous work. It also eliminates the short period effects with periods of one day or less. It seems that only the $k_{2}$ and $k_{3}$ Love numbers and their deviations from constants are of any observational importance. The effects associated with $\mathbf{k}_{4}$, 
$k_{5}, \ldots$ will be damped out by the presence of higher powers of the lunar (solar) parallactic factor.

It must be emphasized that if the deviations of $k_{2}$ and $k_{3}$ from pure constants on the surface of the earth are not taken into consideration, then the danger arises that the perturbations which are caused by the variability of $k_{2}$ and $k_{3}$ will be misinterpreted as the perturbations caused by $k_{4}, \ldots$ Then, the wrong values of $k_{4}, \ldots$ would be obtained.

In the present article, we obtain the expansion of the exterior tidal potential using the theorem of Dirichlet. We also obtain the differential equations for tidal perturbations of elliptic elements taking into account the deviation of $\mathrm{k}_{2}$ and $k_{3}$ from constants along the parallels of latitude.

These differential equations are in a form convenient for numerical integration as well as for the trigonometrical solution (Musen and Estes, 1971), with the arguments $\omega, \delta$ of the satellite and with the standard arguments $\ell, \ell^{\prime}, F$, $D$, and $\Gamma$ of the lunar theory. The short period terms with periods equal to the period of revolution of the satellite or less, were removed from the differential equations by averaging over the instantaneous orbit of the satellite.

A numerical integration and the prediction of the tidal effects is feasible for an interval of a few months.

Using this method, a general idea about very long period influences can be obtained. For example, the influence caused by the rotation of the lunar node can be detected by integration over a number of years.

A full collection of formulas is given to facilitate the programming. 


\section{DISTURBING FUNCTION}

We make use in the present article, similarly as we did in the previous work (Musen and Estes, 1971), of the Maxwellian form of the expansion of the tidal potential into a series of products of spherical harmonics. The first factor in each product is a polynomial in the components $\lambda^{\prime}, \mu^{\prime}, \nu^{\prime}$ of the unit vector directed from the center of the Earth toward the Moon, and the second factor is a polynomial in the analogous quantities $\lambda, \mu, \nu$ for the satellite.

We resort to this representation of the disturbing function, and not to the representation in terms of the elements of the motion of the Moon, because at the present time we do not possess the expansion of the lunar osculating elements into a periodic series with the standard arguments $l, l^{\prime}, F$ and $D$ of the lunar theory. The raised standards of the accuracy of computations do not favor the substitution of the lunar mean elements into the differential equations for the lunar or tidal perturbations of the satellite.

We know, however, the expansions of the rectangular coordinates of the Moon, and consequently also of $\lambda^{\prime}, \mu^{\prime}, \nu^{\prime}$, and their numerical values with an accuracy which permits us to determine the tidal effects of even higher order.

The tidal disturbing function on the surface of the Earth has the form

$$
\Omega=\frac{G m^{\prime}}{a^{\prime 3}}\left(\frac{a^{\prime}}{r^{\prime}}\right)^{3} \sum_{j=0}^{4} A_{j}^{\prime} a_{j}+\frac{G m^{\prime}}{a^{\prime 4}}\left(\frac{a^{\prime}}{r^{\prime}}\right)^{4} \sum_{j=1}^{7} B_{j}^{\prime} b_{j}
$$

where we set 


$$
\begin{aligned}
& \mathrm{A}_{0}^{\prime}=+\frac{1}{4}\left(1-3 \nu^{\prime 2}\right)=-\frac{1}{2} \mathrm{P}_{20}\left(\nu^{\prime}\right), \\
& \mathrm{A}_{1}^{\prime}=+\frac{3}{4}\left(\lambda^{\prime 2}-\mu^{\prime 2}\right)=+\frac{1}{4} \mathrm{P}_{22}\left(\nu^{\prime}\right) \cos 2 \mathrm{~A}^{\prime}, \\
& \mathrm{A}_{2}^{\prime}=+3 \lambda^{\prime} \mu^{\prime}=+\frac{1}{2} \mathrm{P}_{22}\left(\nu^{\prime}\right) \sin 2 \mathrm{~A}^{\prime}, \\
& \mathrm{A}_{3}^{\prime}=+3 \lambda^{\prime} \nu^{\prime}=\mathrm{P}_{21}\left(\nu^{\prime}\right) \cos \mathrm{A}^{\prime}, \\
& \mathrm{A}_{4}^{\prime}=+3 \mu^{\prime} \nu^{\prime}=\mathrm{P}_{21}\left(\nu^{\prime}\right) \sin \mathrm{A}^{\prime} . \\
& \mathrm{B}_{1}^{\prime}=+\frac{3}{8} \lambda^{\prime}\left(1-5 \nu^{\prime 2}\right)=-\frac{1}{4} \mathrm{P}_{31}\left(\nu^{\prime}\right) \cos \mathrm{A}^{\prime}, \\
& \mathrm{B}_{7}^{\prime}=+15 \lambda^{\prime} \mu^{\prime} \nu^{\prime}=+\frac{1}{2} \mathrm{P}_{32}\left(\nu^{\prime}\right) \sin 2 \mathrm{~A}^{\prime}, \\
& \mathrm{B}_{6}^{\prime}=+\frac{3}{8} \mu^{\prime}\left(1-5 \nu^{\prime 2}\right)=-\frac{1}{4} \mathrm{P}_{31}\left(\nu^{\prime}\right) \sin \mathrm{A}^{\prime}, \\
& \mathrm{B}_{3}^{\prime}=+\frac{1}{4} \nu^{\prime}\left(3-5 \nu^{\prime 2}\right)=-\frac{1}{2} \mathrm{P}_{30}\left(\nu^{\prime}\right), \\
& \nu^{\prime}\left(\lambda^{\prime 2}-\mu^{\prime 2}\right)=+\frac{1}{4} \mathrm{P}_{32}\left(\nu^{\prime}\right) \cos 2 \mathrm{~A}^{\prime}, \\
& \left.\mu^{\prime 2}\right)=+\frac{1}{24} \mathrm{P}_{33}\left(\nu^{\prime}\right) \sin 3 \mathrm{~A}^{\prime}, \\
& \left.\lambda^{\prime 2}-3 \mu^{\prime 2}\right)=+\frac{1}{24} \mathrm{P}_{33}\left(\nu^{\prime}\right) \cos 3 \mathrm{~A}^{\prime},
\end{aligned}
$$




$$
\begin{aligned}
& a_{0}=k_{2} r^{2}\left(1-3 \nu^{2}\right)=-2 k_{2} r^{2} P_{20}(\nu) ; \\
& a_{1}=k_{2} r^{2}\left(\lambda^{2}-\mu^{2}\right)=+\frac{1}{3} k_{2} r^{2} P_{22}(\nu) \cos 2 A, \\
& a_{2}=k_{2} r^{2} \lambda \mu=+\frac{1}{6} k_{2} r^{2} P_{22}(\nu) \sin 2 A, \\
& a_{3}=k_{2} r^{2} \lambda \nu=+\frac{1}{3} k_{2} r^{2} P_{21}(\nu) \cos A, \\
& a_{4}=k_{2} r^{2} \mu \nu=+\frac{1}{3} k_{2} r^{2} P_{21}(\nu) \sin A .
\end{aligned}
$$

$$
\mathrm{b}_{1}=\mathrm{k}_{3} \mathrm{r}^{3} \lambda\left(1-5 \nu^{2}\right)=-\frac{2}{3} \mathrm{k}_{3} \mathrm{r}^{3} \mathrm{P}_{31}(\nu) \cos \mathrm{A},
$$$$
b_{2}=k_{3} r^{3} \mu\left(1-5 \nu^{2}\right)=-\frac{2}{3} k_{3} r^{3} P_{31}(\nu) \text { sin } A,
$$$$
b_{3}=k_{3} r^{3} \nu\left(3-5 \nu^{2}\right)=-2 k_{3} r^{3} P_{30}(\nu),
$$$$
b_{4}=k_{3} r^{3} \lambda\left(\lambda^{2}-3 \mu^{2}\right)=+\frac{1}{15} k_{3} r^{3} P_{33}(\nu) \cos 3 A,
$$

$$
b_{5}=k_{3} r^{3} \mu\left(3 \lambda^{2}-\mu^{2}\right)=+\frac{1}{15} k_{3} r^{3} P_{33}(\nu) \sin 3 A,
$$

$$
b_{6}=k_{3} r^{3} \nu\left(\lambda^{2}-\mu^{2}\right)=+\frac{1}{15} k_{3} r^{3} P_{32}(\nu) \cos 2 A
$$

$$
b_{7}=k_{3} r^{3} \lambda \mu \nu=+\frac{1}{30} k_{3} r^{3} P_{32}(\nu) \text { s in } 2 A .
$$

5 
and $\mathrm{k}_{2}, \mathrm{k}_{3}, \ldots$ are the Love numbers. They characterize the local elastic properties of the Earth on its surface. The Love numbers are usually assumed to be constants and their so-called "global" values are substituted into the expansion of the tidal disturbing function. The "global" Love numbers represent the averaged elastic properties of the Earth as they are being observed by the satellite from outer space. The introduction of the global Love numbers yields interesting and important results confirmed by observations. All existing analytical expansions or the numerical integration of the tidal effects consider only $\mathrm{k}_{2}$.

The inclusion of the $\mathrm{k}_{3}$-part, and possibly the higher order parts, represents a more subtle problem and attention must be given to some local properties of Love numbers and also to the deviation of the Earth from a sphere.

We assume, together with Kaula (1969), that the Love numbers are functions smoothly varying on the surface of the Earth and can be expanded into a series of spherical harmonics. We do not know yet the values of coefficients in this expansion, but we can expect that in our problem, because of the averaging process performed by the rotation of the Earth, the dependence of $k_{2}, k_{3}, \ldots$ on the latitude will be stronger than dependence upon the longitude and we can set (Kaula, 1969):

$$
k_{\ell}=k_{\ell 0}+k_{\ell_{1}} P_{10}(\nu)+k_{\ell 2} P_{20}(\nu)+\cdots \cdots .
$$

The neglected terms in this expansion are periodic with a period of one day or less. At the present time they can be considered as short periodic and therefore can be omitted. 
Thus, following Kaula (1969), the averaging process will be performed only along the parallels, and not over the whole Earth. The values of $k_{2}$ as obtained from the observation of satellites by different authors (Kozai, 1965), (Newton, 1968), (Smith, et. al., 1971), (Musen and Estes, 1971) are not in complete agreement. The determination of $\mathrm{k}_{3}$ from the motion of satellites still must be done.

In order to facilitate the selection of the numerically significant part of the tidal potential we computed estimates of the factors attached to the terms in the expansion of this potential. We have the following information on the basis of present knowledge:

$$
\mathrm{k}_{20} \sim 0.3, \mathrm{k}_{30} \sim 0.1, \mathrm{k}_{22} \sim 0.1
$$

and we believe that we are on the safe side if we assume the following preliminary estimates:

$$
\mathrm{k}_{21} \sim 0.01, \mathrm{k}_{31} \sim 0.01, \mathrm{k}_{4} \sim 0.1
$$

Using these numbers we obtain the following estimates for the indicated factors:

$1^{\circ}$. direct $\mathrm{k}_{2}$ part:

$$
\frac{\mathrm{m}^{\prime}}{\mathrm{M}} a^{\prime 3} \mathrm{k}_{20} \sim 1.7 \times 10^{-8},
$$

$2^{\circ}$. direct $\mathrm{k}_{3}$ part:

$$
\frac{m^{\prime}}{M} \alpha^{\prime 4} k_{30} \sim 1 \times 10^{-10}
$$

$3^{\circ}$. direct $\mathrm{k}_{4}$ part:

$$
\frac{\mathrm{m}^{\prime}}{\mathrm{M}} \alpha^{\prime 5} \mathrm{k}_{4} \sim 1.6 \times 10^{-12}
$$


$4^{\circ}$. terms representing the influence on $2^{\circ}$. of the deviation of $k_{2}$ from a constant:

$$
\frac{\mathrm{m}^{\prime}}{\mathrm{M}} \alpha^{\prime 3} \mathrm{k}_{21} \sim 5.6 \times 10^{-10}
$$

$5^{\circ}$. terms representing the influence on $3^{\circ}$ of the deviation of $k_{2}$ from a constant:

$$
\frac{\mathrm{m}^{\prime}}{\mathrm{M}} a^{\prime 3} \mathrm{k}_{22} \sim 5.6 \times 10^{-9}
$$

$6^{\circ}$. terms representing the influence on $3^{\circ}$ of the deviation of $k_{3}$ from a constant:

$$
\frac{\mathrm{m}^{\prime}}{\mathrm{M}} a^{\prime 3} \mathrm{k}_{31} \sim 5.6 \times 10^{-10}
$$

$7^{\circ}$. terms representing the influence of the oblateness on $2^{\circ}$ :

$$
\frac{\mathrm{m}^{\prime}}{\mathrm{M}} a^{\prime 4} \mathrm{k}_{20} \epsilon^{2} \sim 1.9 \times 10^{-12}
$$

$8^{\circ}$. terms representing the influence of the oblateness on $3^{\circ}$ :

$$
\frac{\mathrm{m}^{\prime}}{\mathrm{M}} a^{\prime 3} \mathrm{k}_{20} \epsilon^{2} \sim 1.2 \times 10^{-10}
$$

We see from this table that the part $3^{\circ}$ associated with $\mathrm{k}_{4}$ constitutes only $1 \%$ of the terms $8^{\circ}$ having the same trigonometrical arguments as $3^{\circ}$ and $\mathrm{k}_{20} \epsilon^{2}$ as a factor. 
Also, we maintain that the terms having $k_{s}(s>3)$ as a factor will be less influential than the terms having the same trigonometrical arguments and factors of the type $k_{20} \times k_{2 j}, k_{30} \times k_{3 j}$.

In this case it will be difficult to determine $k_{s}(s>3)$ Love numbers. The parts of the tidal potential having $\mathrm{k}_{2}$ and $\mathrm{k}_{3}$ as factors will be the parts which have dominant physical and numerical significance in the theory of tidal perturbations of close satellites.

For this reason, we include in the disturbing function terms of the types $1^{\circ}, 2^{\circ}, 4^{\circ}, 5^{\circ}, 6^{\circ}$ and $8^{\circ}$, and omit the terms $3^{\circ}$ and $7^{\circ}$.

Substituting

$$
\begin{aligned}
& k_{2}=k_{20}+k_{21} P_{10}(\nu)+k_{22} P_{20}(\nu) \\
& k_{3}=k_{30}+k_{31} P_{10}(\nu)+\cdots
\end{aligned}
$$

into (3) and $\left(3^{\prime}\right)$, retaining only the significant terms and redeveloping the results in terms of spherical harmonics in form convenient for the continuation of the potential into outer space, we obtain

$$
\begin{aligned}
a_{0}= & r^{2}\left[-\frac{2}{5} k_{22}-\frac{4}{5} k_{21} \nu+\left(k_{20}+\frac{2}{7} k_{22}\right)\left(1-3 \nu^{2}\right)\right. \\
& \left.+\frac{3}{5} k_{21} \nu\left(3-5 \nu^{2}\right)-\frac{9}{70} k_{22}\left(3-30 \nu^{2}+35 \nu^{4}\right)\right],
\end{aligned}
$$




$$
\begin{aligned}
& a_{1}=r^{2}\left(\lambda^{2}-\mu^{2}\right)\left[+\left(k_{20}-\frac{2}{7} k_{22}\right)+k_{21} \nu-\frac{3}{4} k_{22}\left(1-7 \nu^{2}\right)\right], a_{2}=r^{2} \lambda \mu\left[+\left(k_{20}-\frac{2}{7} k_{22}\right)+k_{21} \nu-\frac{3}{14} k_{22}\left(1-7 \nu^{2}\right)\right] \\
& a_{3}=r^{2} \lambda\left[+\frac{1}{5} k_{21}+\left(k_{20}+\frac{1}{7} k_{22}\right) \nu-\frac{1}{5} k_{21}\left(1-5 \nu^{2}\right)\right. \\
&\left.-\frac{9}{14} k_{22} \nu\left(1-\frac{7}{3} \nu^{2}\right)\right], \\
& {\left[+\frac{1}{5} k_{21}+\left(k_{20}+\frac{1}{7} k_{22}\right) \nu-\frac{1}{5} k_{21}\left(1-5 \nu^{2}\right)\right.} \\
& a_{4}=r^{2} \mu\left.-\frac{9}{14} k_{22} \nu\left(1-\frac{7}{3} \nu^{2}\right)\right]
\end{aligned}
$$

and

$$
\begin{aligned}
& \mathrm{b}_{1}=\mathrm{r}^{3} \lambda\left[-\frac{8}{7} \mathrm{k}_{31} \nu+\mathrm{k}_{30}\left(1-5 \nu^{2}\right)+\frac{15}{7} \mathrm{k}_{31} \nu\left(1-\frac{7}{3} \nu^{2}\right)\right] \\
& \mathrm{b}_{2}=\mathrm{r}^{3} \mu\left[-\frac{8}{7} \mathrm{k}_{31} \nu+\mathrm{k}_{30}\left(1-5 \nu^{2}\right)+\frac{15}{7} \mathrm{k}_{31} \nu\left(1-\frac{7}{3} \nu^{2}\right)\right] \\
& \mathrm{b}_{3}=\mathrm{r}^{3}\left[+\frac{3}{7} \mathrm{k}_{31}\left(1-3 \nu^{2}\right)+\mathrm{k}_{30} \nu\left(3-5 \nu^{2}\right)-\frac{1}{7} \mathrm{k}_{31}\left(3-30 \nu^{2}+35 \nu^{4}\right)\right], \\
& \mathrm{b}_{4}=\mathrm{r}^{3} \lambda\left(\lambda^{2}-3 \mu^{2}\right)\left(\mathrm{k}_{30}+\mathrm{k}_{31} \nu\right), \\
& \mathrm{b}_{5}=\mathrm{r}^{3} \mu\left(3 \lambda^{2}-\mu^{2}\right)\left(\mathrm{k}_{30}+\mathrm{k}_{31} \nu\right), \\
& \mathrm{b}_{6}=\mathrm{r}^{3}\left(\lambda^{2}-\mu^{2}\right)\left[+\frac{1}{7} \mathrm{k}_{31}+\mathrm{k}_{30} \nu-\frac{1}{7} \mathrm{k}_{31}\left(1-7 \nu^{2}\right)\right] \\
& \mathrm{b}_{7}=\mathrm{r}^{3} \lambda \mu\left[+\frac{1}{7} \mathrm{k}_{31}+\mathrm{k}_{30} \nu-\frac{1}{7}\left(1-7 \nu^{2}\right)\right] .
\end{aligned}
$$

10 
For the extensions of $a_{j}$ and $b_{j}$ into outer space we have with the same accuracy as before:

$$
\begin{aligned}
A_{0}= & {\left[\frac{2}{3} \epsilon^{2}\left(k_{20}+\frac{2}{7} k_{22}\right)-\frac{2}{5} k_{22}\right] \frac{a^{\prime 3}}{a} a^{\prime 3}\left(\frac{a}{r}\right) } \\
& -\frac{4}{5} k_{21} \frac{a^{\prime 3}}{a} a a^{\prime 3}\left(\frac{a}{r}\right)^{2} \nu \\
& +\left(k_{20}+\frac{2}{7} k_{22}\right)\left(1-\frac{55}{42} \epsilon^{2}\right) \frac{a^{\prime 3}}{a} a^{2} a^{\prime 3}\left(\frac{a}{r}\right)^{3}\left(1-3 \nu^{2}\right) \\
& +\frac{3}{5} k_{21} \frac{a^{\prime 3}}{a} a^{3} a^{\prime 3}\left(\frac{a}{r}\right)^{4}\left(3 \nu-5 \nu^{3}\right) \\
& +\left[\frac{3}{14} \epsilon^{2}\left(k_{20}+\frac{2}{7} k_{22}\right)-\frac{9}{70} k_{22}\right] \frac{a^{\prime 3}}{a} a^{4} a^{\prime 3}\left(\frac{a}{r}\right)^{5}\left(3-30 \nu^{2}+35 \nu^{4}\right), \\
A_{1}= & \left(k_{20}-\frac{2}{7} k_{22}\right)\left(1-\frac{5}{14} \epsilon^{2}\right) \frac{a^{\prime 3}}{a} a^{2} a^{\prime 3}\left(\frac{a}{r}\right)^{3}\left(\lambda^{2}-\mu^{2}\right), \\
& +\left[\frac{5}{14} \epsilon^{2}\left(k_{20}-\frac{2}{7} k_{22}\right)-\frac{3}{4} k_{22}\right] \frac{a^{\prime 3}}{a} a^{4} a^{\prime 3}\left(\frac{a}{r}\right)^{5} \lambda \mu\left(1-7 \nu^{2}\right), \\
A_{2}= & \left(k_{20}-\frac{2}{7} k_{22}\right)\left(1-\frac{5}{14} \epsilon^{2}\right) \frac{a^{\prime 3}}{a} a^{2} a^{\prime 3}\left(\frac{a}{r}\right)^{3} \lambda \mu \\
& +k_{21} \frac{a^{\prime 3}}{a} a^{3} a^{\prime 3}\left(\frac{a}{r}\right)^{4} \nu\left(\lambda^{2}-\mu^{2}\right) \\
& +\left[\frac{5}{14} \epsilon^{2}\left(k_{20}-\frac{2}{7} k_{22}\right)-\frac{3}{4} k_{22}\right] \frac{a^{\prime 3}}{a} a^{4} a^{\prime 3}\left(\frac{a}{r}\right)^{5}\left(\lambda^{2}-\mu^{2}\right)\left(1-7 \nu^{2}\right), \\
& +4 \nu
\end{aligned}
$$




$$
\begin{aligned}
& A_{3}=+\frac{1}{5} k_{21} \frac{a^{3}}{a} \alpha \alpha^{\prime 3}\left(\frac{a}{r}\right)^{2} \lambda \\
& +\left(\mathrm{k}_{20}+\frac{1}{7} \mathrm{k}_{22}\right)\left(1-\frac{15}{14} \epsilon^{2}\right) \frac{\mathrm{a}^{\prime 3}}{\mathrm{a}} \alpha^{2} \alpha^{\prime 3}\left(\frac{\mathrm{a}}{\mathrm{r}}\right)^{3} \lambda \nu \\
& -\frac{1}{5} k_{21} \frac{a^{\prime 3}}{a} a^{3} a^{\prime 3}\left(\frac{a}{r}\right)^{4} \lambda\left(1-5 \nu^{2}\right) \\
& +\left[\frac{15}{14} \epsilon^{2}\left(\mathrm{k}_{20}+\frac{1}{7} \mathrm{k}_{22}\right)-\frac{9}{14} \mathrm{k}_{22}\right] \frac{\mathrm{a}^{33}}{\mathrm{a}} \alpha^{4} a^{\prime 3}\left(\frac{\mathrm{a}}{\mathrm{r}}\right)^{5} \lambda \nu\left(1-\frac{7}{3} \nu^{2}\right), \\
& A_{4}=+\frac{1}{5} k_{21} \frac{a^{\prime 3}}{a} \alpha \alpha^{\prime 3}\left(\frac{a}{r}\right)^{2} \mu \\
& +\left(\mathrm{k}_{20}+\frac{1}{7} \mathrm{k}_{22}\right)\left(1-\frac{1.5}{14} \epsilon^{2}\right) \frac{\mathrm{a}^{3}}{\mathrm{a}} \alpha^{2} \alpha^{\prime 3}\left(\frac{\mathrm{a}}{\mathrm{r}}\right)^{3} \mu \nu \\
& -\frac{1}{5} \mathrm{k}_{21} \frac{\mathrm{a}^{\prime 3}}{\mathrm{a}} \alpha^{3} a^{\prime 3}\left(\frac{\mathrm{a}}{\mathrm{r}}\right)^{4} \mu\left(1-5 \nu^{2}\right) \\
& +\left[\frac{15}{14} \epsilon^{2}\left(\mathrm{k}_{20}+\frac{1}{7} \mathrm{k}_{22}\right)-\frac{9}{14} \mathrm{k}_{22}\right] \frac{\mathrm{a}^{\prime 3}}{\mathrm{a}} \alpha^{4} \alpha^{\prime 3}\left(\frac{\mathrm{a}}{\mathrm{r}}\right)^{5} \mu \nu\left(1-\frac{7}{3} \nu^{2}\right), \\
& \mathrm{B}_{1}=-\frac{8}{7} \mathrm{k}_{31} \frac{\mathrm{a}^{\prime 4}}{\mathrm{a}} \alpha^{2} \alpha^{.4}\left(\frac{\mathrm{a}}{\mathrm{r}}\right)^{3} \lambda \nu \\
& +k_{30} \frac{a^{\prime 4}}{a} \alpha^{3} \alpha^{\prime 4}\left(\frac{a}{r}\right)^{4} \lambda\left(1-5 \nu^{2}\right) \\
& +\frac{15}{7} \mathrm{k}_{31} \frac{\mathrm{a}^{\prime 4}}{\mathrm{a}} \alpha^{4} \alpha^{64}\left(\frac{\mathrm{a}}{\mathrm{r}}\right)^{5} \lambda \nu\left(1-\frac{7}{3} \nu^{2}\right),
\end{aligned}
$$

12 


$$
\begin{aligned}
& \mathrm{B}_{2}=-\frac{8}{7} \mathrm{k}_{31} \frac{\mathrm{a}^{\prime 4}}{\mathrm{a}} \alpha^{2} a^{\prime 4}\left(\frac{\mathrm{a}}{\mathrm{r}}\right)^{3} \mu \nu \\
& +k_{30} \frac{a^{\prime 4}}{a} a^{3} a^{\prime 4}\left(\frac{a}{r}\right)^{4} \mu\left(1-5 \nu^{2}\right) \\
& +\frac{15}{7} k_{31} \frac{a^{\prime 4}}{a} a^{4} a^{\prime 4}\left(\frac{a}{r}\right)^{5} \mu \nu\left(1-\frac{7}{3} \nu^{2}\right), \\
& \mathrm{B}_{3}=+\frac{3}{7} \mathrm{k}_{31} \frac{\mathrm{a}^{\prime 4}}{\mathrm{a}} a^{2} \alpha^{\prime 4}\left(\frac{\mathrm{a}}{\mathrm{r}}\right)^{3}\left(1-3 \nu^{2}\right) \\
& +k_{30} \frac{a^{\prime 4}}{a} \alpha^{3} \alpha^{\prime 4}\left(\frac{a}{r}\right)^{4}\left(3 \nu-5 \nu^{3}\right) \\
& -\frac{1}{7} k_{31} \frac{a^{14}}{a} \alpha^{4} \alpha^{\prime 4}\left(\frac{a}{r}\right)^{5}\left(3-30 \nu^{2}+35 \nu^{4}\right) \text {, } \\
& B_{4}=k_{30} \frac{a^{\prime 4}}{a} a^{3} a^{\prime 4}\left(\frac{a}{r}\right)^{4} \lambda\left(\lambda^{2}-3 \mu^{2}\right) \\
& +k_{31} \frac{a^{\prime 4}}{a} a^{4} \alpha^{\prime 4}\left(\frac{a}{r}\right)^{5} \lambda \nu\left(\lambda^{2}-3 \mu^{2}\right), \\
& \mathrm{B}_{5}=\mathrm{k}_{30} \frac{\mathrm{a}^{\prime 4}}{\mathrm{a}} a^{3} a^{\prime 4}\left(\frac{\mathrm{a}}{\mathrm{r}}\right)^{4} \mu\left(3 \lambda^{2}-\mu^{2}\right) \\
& +k_{31} \frac{a^{\prime 4}}{a} a^{4} \alpha^{\prime 4}\left(\frac{a}{r}\right)^{5} \mu \nu\left(3 \lambda^{2}-\mu^{2}\right),
\end{aligned}
$$




$$
\begin{aligned}
\mathrm{B}_{6}= & +\frac{1}{7} \mathrm{k}_{31} \frac{\mathrm{a}^{4}}{\mathrm{a}} \alpha^{2} \alpha^{\prime 4}\left(\frac{\mathrm{a}}{\mathrm{r}}\right)^{3}\left(\lambda^{2}-\mu^{2}\right) \\
& +\mathrm{k}_{30} \frac{\mathrm{a}^{\prime 4}}{\mathrm{a}} a^{3} \alpha^{\prime 4}\left(\frac{\mathrm{a}}{\mathrm{r}}\right)^{4} \nu\left(\lambda^{2}-\mu^{2}\right) \\
& -\frac{1}{7} \mathrm{k}_{31} \frac{\mathrm{a}^{\prime 4}}{\mathrm{a}} a^{4} \alpha^{\prime 4}\left(\frac{\mathrm{a}}{\mathrm{r}}\right)^{5}\left(\lambda^{2}-\mu^{2}\right)\left(1-7 \nu^{2}\right), \\
\mathrm{B}_{7}= & +\frac{1}{7} \mathrm{k}_{31} \frac{\mathrm{a}^{\prime 4}}{\mathrm{a}} a^{2} \alpha^{\prime 4}\left(\frac{\mathrm{a}}{\mathrm{r}}\right)^{3} \lambda \mu \\
& +\mathrm{k}_{30} \frac{\mathrm{a}^{\prime 4}}{\mathrm{a}} a^{3} \alpha^{\prime 4}\left(\frac{\mathrm{a}}{\mathrm{r}}\right)^{4} \lambda \mu \nu \\
& -\frac{1}{7} \mathrm{k}_{31} \frac{\mathrm{a}^{14}}{\mathrm{a}} \alpha^{4} \alpha^{14}\left(\frac{\mathrm{a}}{\mathrm{r}}\right)^{5} \lambda \mu\left(1-7 \nu^{2}\right),
\end{aligned}
$$

It can be checked easily that in outer space $A_{j}$ and $B_{j}$ satisfy the Laplace differential equation. On the surface of the Earth, using

$$
\frac{\mathrm{R}^{2}}{\mathrm{r}^{2}}=1+\epsilon^{2} \nu^{2}+\cdots \cdot
$$

$A_{j}$ and $B_{j}$ become, respectively, $a_{j}$ and $b_{j}$. These expansions represent $a$ generalization of the expansions obtained previously (Musen and Estes, 1971) where the assumption is made that $\mathrm{k}_{2}$ and $\mathrm{k}_{3}$ are constants.

The terms of higher order in $\epsilon^{2}$ are not of any importance from the observational standpoint and they are therefore omitted. 
Substituting (5) and (5') into (1) instead of $a_{j}(j=0,1, \ldots, 4)$ and $b_{j}(j=$ $1,2, \ldots, 7)$ and making use of

$$
G M=n^{2} a^{3}
$$

we obtain the following representation of the tidal disturbing function in outer space:

$$
\begin{aligned}
\Omega= & \frac{m^{\prime}}{M} a^{\prime 3}\left(\frac{2}{3} \epsilon^{2}-\frac{2}{5} k_{22}\right)\left(\frac{a^{\prime}}{r^{\prime}}\right)^{3} A_{0}^{\prime} \cdot \frac{G M}{r} \\
& +n^{2} a^{2} \frac{m^{\prime}}{M} a a^{\prime 3} k_{21}\left(\frac{a^{\prime}}{r^{\prime}}\right)^{3}\left(\frac{a}{r}\right)^{2} V_{1} \\
& +n^{2} a^{2} \frac{m^{\prime}}{M} a^{2} a^{\prime 3}\left(\frac{a^{\prime}}{r^{\prime}}\right)^{3}\left(\frac{a}{r}\right)^{3} V_{2} \\
& +n^{2} a^{2} \frac{m^{\prime}}{M} a^{3} a^{\prime 3}\left(\frac{a^{\prime}}{r^{\prime}}\right)^{3}\left(\frac{a}{r}\right)^{4} V_{3} \\
& +n^{2} a^{2} \frac{m^{\prime}}{M} a^{4} a^{\prime 3}\left(\frac{a^{\prime}}{r^{\prime}}\right)^{3}\left(\frac{a}{r}\right)^{5} V_{4} .
\end{aligned}
$$

$\mathrm{V}_{2}$ is the dominant part. It defines the so-called "main problem." It is of the second degree in $\lambda, \mu$ and $\nu$. We have

$$
\mathrm{V}_{2}=\mathrm{P}_{0}^{\prime} \mathrm{P}_{0}+\mathrm{P}_{1}^{\prime} \mathrm{P}_{1}+\mathrm{P}_{2}^{\prime} \mathrm{P}_{2}+\mathrm{P}_{3}^{\prime} \mathrm{P}_{3}+\mathrm{P}_{4}^{\prime} \mathrm{P}_{4}
$$

where

$$
\begin{gathered}
\mathrm{P}_{0}^{\prime}=\left(\mathrm{k}_{20}+\frac{2}{7} \mathrm{k}_{22}\right)\left(1-\frac{55}{42} \epsilon^{2}\right) \mathrm{A}_{0}^{\prime}+\frac{3}{7} \mathrm{k}_{31} a^{\prime} \frac{\mathrm{a}^{\prime}}{\mathrm{r}^{\prime}} \mathrm{B}_{3}^{\prime}, \\
\mathrm{P}_{0}=1-3 \nu^{2},
\end{gathered}
$$




$$
\begin{aligned}
& \mathrm{P}_{1}^{\prime}=\left(\mathrm{k}_{20}-\frac{2}{7} \mathrm{k}_{22}\right)\left(1-\frac{5}{14} \epsilon^{2}\right) \mathrm{A}_{1}^{\prime}+\frac{1}{7} \mathrm{k}_{31} a^{\prime} \frac{\mathrm{a}^{\prime}}{\mathrm{r}^{\prime}} \mathrm{B}_{6}^{\prime}, \\
& \mathbf{P}_{1}=\lambda^{2}-\mu^{2} \\
& P_{2}^{\prime}=\left(k_{20}-\frac{2}{7} k_{22}\right)\left(1-\frac{5}{14} \epsilon^{2}\right) A_{2}^{\prime}+\frac{1}{7} k_{31} a^{\prime} \frac{a^{\prime}}{r^{\prime}} B_{7}^{\prime}, \\
& \mathbf{P}_{2}=\lambda \mu \text {, } \\
& \mathrm{P}_{3}^{\prime}=\left(\mathrm{k}_{20}+\frac{1}{7} \mathrm{k}_{22}\right)\left(1-\frac{15}{14} \epsilon^{2}\right) \mathrm{A}_{3}^{\prime}-\frac{8}{7} \mathrm{k}_{31} a^{\prime} \frac{\mathrm{a}^{\prime}}{\mathrm{r}^{\prime}} \mathrm{B}_{1}^{\prime}, \\
& \mathbf{P}_{3}=\lambda \nu, \\
& \mathrm{P}_{4}^{\prime}=\left(\mathrm{k}_{20}+\frac{1}{7} \mathrm{k}_{22}\right)\left(1-\frac{15}{14} \epsilon^{2}\right) \mathrm{A}_{4}^{\prime}-\frac{8}{7} \mathrm{k}_{31} a^{\prime} \frac{\mathrm{a}^{\prime}}{\mathrm{r}^{\prime}} \mathrm{B}_{2}^{\prime}, \\
& \mathbf{P}_{4}=\mu \nu \text {. }
\end{aligned}
$$

The term $V_{3}$ is of the third degree in $\lambda, \mu$ and $\nu$. It contains the $k_{30}$ part and the influence of the deviation of $k_{2}$ from a constant.

$$
V_{3}=S_{1}^{\prime} S_{1}+S_{2}^{\prime} S_{2}+S_{3}^{\prime} S_{3}+S_{4}^{\prime} S_{4}+S_{5}^{\prime} S_{5}+S_{6}^{\prime} S_{6}+S_{7}^{\prime} S_{7}
$$

where

$$
\begin{gathered}
S_{1}^{\prime}=-\frac{1}{5} k_{21} A_{3}^{\prime}+k_{30} a^{\prime} \frac{a^{\prime}}{r^{\prime}} \cdot B_{1}^{\prime}, \\
S_{1}=\lambda\left(1-5 \nu^{2}\right),
\end{gathered}
$$




$$
\begin{gathered}
S_{2}^{\prime}=-\frac{1}{5} k_{21} A_{4}^{\prime}+k_{30} a^{\prime} \frac{a^{\prime}}{r^{\prime}} B_{2}^{\prime}, \\
S_{2}=\mu\left(1-5 \nu^{2}\right), \\
S_{3}^{\prime}=+\frac{3}{5} k_{21} A_{0}^{\prime}+k_{30} a^{\prime} \frac{a^{\prime}}{r^{\prime}} B_{3}^{\prime}, \\
S_{3}=\nu\left(3-5 \nu^{2}\right), \\
S_{4}^{\prime}=k_{21} A_{2}^{\prime}+k_{30} a^{\prime} \frac{a^{\prime}}{r^{\prime}} B_{7}^{\prime}, \\
S_{7}=\lambda \mu \nu . \\
S_{6}^{\prime}=+k_{21}^{\prime} B_{4}^{\prime}, \\
S_{5}^{\prime}=k_{30} a^{\prime} \frac{a^{\prime}}{r^{\prime}} B_{5}^{\prime}, \\
S_{5}=\lambda\left(\lambda_{30} a^{\prime} \frac{a^{\prime}}{r^{\prime}} B_{6}^{\prime},\right. \\
S^{\prime},
\end{gathered}
$$


$V_{4}$ contains the influence of the oblateness and also the influence of the

deviation of $k_{2}$ and $k_{3}$ from constants. It is of the fourth degree in $\lambda, \mu$ and $\nu$. We have

$$
\mathrm{V}_{4}=\mathrm{T}_{1}^{\prime} \mathrm{T}_{1}+\mathrm{T}_{2}^{\prime} \mathrm{T}_{2}+\mathrm{T}_{3}^{\prime} \mathrm{T}_{3}+\mathrm{T}_{4}^{\prime} \mathrm{T}_{4}+\mathrm{T}_{5}^{\prime} \mathrm{T}_{5}+\mathrm{T}_{6}^{\prime} \mathrm{T}_{6}+\mathrm{T}_{7}^{\prime} \mathrm{T}_{7},
$$

where

$$
\begin{gathered}
\mathrm{T}_{1}^{\prime}=\left[+\frac{15}{14} \epsilon^{2}\left(\mathrm{k}_{20}+\frac{1}{7} \mathrm{k}_{22}\right)-\frac{9}{14} \mathrm{k}_{22}\right] \mathrm{A}_{3}^{\prime}+\frac{15}{7} \mathrm{k}_{31} a^{\prime} \frac{\mathrm{a}^{\prime}}{\mathrm{r}^{\prime}} \mathrm{B}_{1}^{\prime}, \\
\mathrm{T}_{1}=\lambda \nu\left(1-\frac{7}{3} \nu^{2}\right), \\
\mathrm{T}_{2}^{\prime}=\left[\begin{array}{c}
\left.+\frac{15}{14} \epsilon^{2}\left(\mathrm{k}_{20}+\frac{1}{7} \mathrm{k}_{22}\right)-\frac{9}{14} \mathrm{k}_{22}\right] \mathrm{A}_{4}^{\prime}+\frac{15}{7} \mathrm{k}_{31} a^{\prime} \frac{\mathrm{a}^{\prime}}{\mathrm{r}^{\prime}} \mathrm{B}_{2}^{\prime}, \\
\mathrm{T}_{2}=\mu \nu\left(1-\frac{7}{3} \nu^{2}\right), \\
\mathrm{T}_{5}^{\prime}=\mathrm{k}_{31} \mathrm{a}^{\prime} \frac{\mathrm{a}^{\prime}}{\mathrm{r}^{\prime}} \mathrm{B}_{5}^{\prime}, \quad \\
\left.+\frac{3}{14}\left(\mathrm{k}_{20}+\frac{2}{7} \mathrm{k}_{22}\right) \epsilon^{2}-\frac{9}{70} \mathrm{k}_{22}\right] \mathrm{A}_{0}^{\prime}-\frac{1}{7} \mathrm{k}_{31} a^{\prime} \frac{\mathrm{a}^{\prime}}{\mathrm{r}^{\prime}} \mathrm{B}_{3}^{\prime}, \\
\mathrm{T}_{4}^{\prime}=\mathrm{k}_{31} \alpha^{\prime} \frac{\mathrm{a}^{\prime}}{\mathrm{r}^{\prime}} \mathrm{B}_{4}^{\prime}, \\
\mathrm{T}_{3}=3-30 \nu^{2}+35 \nu^{4},
\end{array}\right.
\end{gathered}
$$




$$
\begin{gathered}
\mathrm{T}_{5}=\mu \nu\left(3 \lambda^{2}-\mu^{2}\right), \\
\mathrm{T}_{6}^{\prime}=\left[+\frac{5}{14}\left(\mathrm{k}_{20}-\frac{2}{7} \mathrm{k}_{22}\right) \epsilon^{2}-\frac{3}{4} \mathrm{k}_{22}\right] \mathrm{A}_{1}^{\prime}-\frac{1}{7} \mathrm{k}_{31} a^{\prime} \frac{\mathrm{a}^{\prime}}{\mathrm{r}^{\prime}} \mathrm{B}_{6}^{\prime}, \\
\mathrm{T}_{7}^{\prime}=\left[\begin{array}{c}
\left.\frac{5}{14} \epsilon^{2}\left(\mathrm{k}_{20}-\frac{2}{7} \mathrm{k}_{22}\right)-\frac{3}{4} \mathrm{k}_{22}\right] \mathrm{A}_{2}^{\prime}-\frac{1}{7} \mathrm{k}_{31} \alpha^{\prime} \frac{\mathrm{a}^{\prime}}{\mathrm{r}^{\prime}} \mathrm{B}_{7}^{\prime}, \\
\mathrm{T}_{7}=\lambda \mu\left(1-7 \nu^{2}\right),
\end{array}\right.
\end{gathered}
$$

The factor $\left(a^{\prime} / r^{\prime}\right)^{3} A_{0}^{\prime}$ attached to $G M / r$ in the first term in (1) contains the purely periodic terms with the arguments $\ell, \ell^{\prime}, F, D$ and $\Gamma$ and a constant term. Consequently, the influence of the oblateness and the deviation of $\mathbf{k}_{2}$ from a constant on the tidal potential may affect the accurate determination of GM.

$$
V_{1}=-\frac{4}{5} A_{0}^{\prime} \nu+\frac{1}{5}\left(A_{3}^{\prime} \lambda+A_{4}^{\prime} \mu\right)
$$

The $V_{1}$ terms are not of any importance in the computation of the tidal effect in the motion of an artifical satellite because they disappear in the proces of elimination of the short period terms by averaging the disturbing function over the orbit of the satellite. 
Substituting

$$
\begin{aligned}
& \lambda=+\cos \delta \cos (f+\omega)-\sin \delta \sin (f+\omega) \cos i, \\
& \mu=+\sin \delta \cos (f+\omega)+\cos \delta \sin (f+\omega) \cos i, \\
& \nu=\sin (f+\omega) \sin i
\end{aligned}
$$

into the expressions $\left(7^{\prime}\right)$ for $P_{0}, P_{1}, \ldots$, we obtain

$$
\begin{aligned}
& P_{0}=\left(-\frac{1}{2}+\frac{3}{2} \cos ^{2} i\right)+\frac{3}{2} \sin ^{2} i \cos (2 f+2 \omega) \\
& P_{1}=+\frac{1}{2} \cos 2 \delta \sin ^{2} i+\frac{1}{2} \cos 2 \delta\left(1+\cos ^{2} i\right) \cos (2 f+2 \omega) \\
& -\sin 2 \delta \cos i \sin (2 f+2 \omega) \text {, } \\
& P_{2}=+\frac{1}{4} \sin 2 \delta \sin ^{2} i+\frac{1}{4} \sin 2 \delta b\left(1+\cos ^{2} i\right) \cos (2 f+2 \omega) \\
& +\frac{1}{2} \cos 2 \Omega \cos \text { i sin }(2 f+2 \omega) \text {, } \\
& P_{3}=-\frac{1}{2} \sin \delta \sin i \cos i+\frac{1}{2} \sin \delta \sin i \cos i \cos (2 f+2 \omega) \\
& +\frac{1}{2} \cos \delta \sin i \sin (2 f+2 \omega) \text {, } \\
& \mathrm{P}_{4}=+\frac{1}{2} \cos \delta \cos \mathrm{i} \sin \mathrm{i}-\frac{1}{2} \cos \delta \cos i \sin \mathrm{i} \cos (2 \mathrm{f}+2 \omega) \\
& +\frac{1}{2} \sin \delta b \sin i \sin (2 f+2 \omega)
\end{aligned}
$$


and $V_{2}$ takes the form

$$
V_{2}=V_{20}+V_{22}^{(c)} \cos (2 f+2 \omega)+V_{22}^{(s)} \sin (2 f+2 \omega),
$$

where

$$
\begin{aligned}
& V_{20}=\left(-\frac{1}{2}+\frac{3}{2} \cos ^{2} i\right) K_{20}^{(c)}-\frac{1}{4} K_{21}^{(c)} \sin 2 i+\frac{1}{2} K_{22}^{(c)} \sin ^{2} i \\
& V_{22}^{(c)}=+\frac{3}{2} K_{20}^{(c)} \sin ^{2} i+\frac{1}{4} K_{21}^{(c)} \sin 2 i+\frac{1}{2}\left(1+\cos ^{2} i\right) K_{22}^{(c)}, \\
& V_{22}^{(s)}=+\frac{1}{2} K_{21}^{(s)} \sin i-K_{22}^{(s)} \cos i,
\end{aligned}
$$

where we set:

$$
\begin{aligned}
& \mathrm{K}_{20}^{(c)}=\mathrm{P}_{0}^{\prime}, \\
& \mathrm{K}_{21}^{(\mathrm{c})}=+\mathrm{P}_{3}^{\prime} \sin \delta-\mathrm{P}_{4}^{\prime} \cos \delta, \\
& \mathrm{K}_{22}^{(\mathrm{c})}=+\mathrm{P}_{1}^{\prime} \cos 2 \delta+\frac{1}{2} \mathrm{P}_{2}^{\prime} \sin 2 \delta_{1} \\
& \mathrm{~K}_{21}^{(s)}=+\mathrm{P}_{3}^{\prime} \cos \delta+\mathrm{P}_{4}^{\prime} \sin \delta_{6}, \\
& \mathrm{~K}_{22}^{(s)}=+\mathrm{P}_{1}^{\prime} \sin 2 \delta b-\frac{1}{2} \mathrm{P}_{2}^{\prime} \cos 2 \delta .
\end{aligned}
$$

We also need the expansion of

$$
\mathbf{R} \cdot \nabla_{0} \Omega=+\frac{\partial \Omega}{\partial \lambda} \sin i \sin \Omega-\frac{\partial \Omega}{\partial \mu} \sin i \cos \Omega+\frac{\partial \Omega}{\partial \nu} \cos i,
$$


because it enters into the differential equations for the variation of $\delta$ and $i$. From $\left(7^{\dagger}\right)$ and making use of $(10)$ we deduce:

$$
\begin{aligned}
& R \cdot \nabla_{0} P_{0}=-3 \sin 2 \text { i sin }(f+\omega) \text {, } \\
& \text { R. } \nabla_{0} P_{1}=+2 \sin 2 \beta \sin i \cos (f+\omega)+\cos 2 \Omega \sin 2 \text { i s in }(f+\omega) \text {, } \\
& \mathbf{R} \cdot \nabla_{0} P_{2}=-\cos 2 \delta \sin i \cos (f+\omega)+\frac{1}{2} \sin 2 \delta \sin 2 \text { i s in }(f+\omega) \text {, } \\
& \text { R. } \nabla_{0} P_{3}=+\cos \Omega \cos i \cos (f+\omega)-\sin \delta \cos 2 \text { i sin }(f+\omega) \text {, } \\
& \mathbf{R} \cdot \nabla_{0} \mathrm{P}_{4}=+\sin \delta \cos \mathrm{i} \cos (f+\omega)+\cos \delta \cos 2 \text { i sin }(f+\omega) \text {, }
\end{aligned}
$$

and as a result:

$$
\mathbf{R} \cdot \nabla_{0} V_{2}=+V_{21}^{(c)} \cos (f+\omega)+V_{21}^{(s)} \sin (f+\omega)
$$

where:

$$
\begin{aligned}
& V_{21}^{(c)}=+K_{21}^{(s)} \cos i+2 K_{22}^{(s)} \text { sin } i \\
& V_{21}^{(s)}=-3 K_{20}^{(c)} \sin 2 i-K_{21}^{(c)} \cos 2 i+K_{22}^{(c)} \sin 2 i .
\end{aligned}
$$

In a similar manner, substituting (10) into $\left(8^{\prime}\right)$ we deduce:

$$
\begin{aligned}
S_{1}= & -\frac{1}{4}\left(1-5 \cos ^{2} i\right) \cos \delta \cos (f+\omega)+\frac{1}{4}\left(11-15 \cos ^{2} i\right) \cos i \sin \delta \sin (f+\omega) \\
& +\frac{5}{4} \sin ^{2} i \cos \delta \cos (3 f+3 \omega)-\frac{5}{4} \sin ^{2} i \cos \text { i s in } \delta \sin (3 f+3 \omega),
\end{aligned}
$$


$S_{2}=-\frac{1}{4}\left(1-5 \cos ^{2} i\right) \sin \Omega \cos (f+\omega)-\frac{1}{4}\left(11-15 \cos ^{2} i\right) \cos i \cos \Omega \sin (f+\omega)$

$+\frac{5}{4} \sin ^{2} i \sin 8 \cos (3 f+3 \omega)+\frac{5}{4} \sin ^{2} i \cos i \cos 8 \sin (3 f+3 \omega)$

$S_{3}=-\frac{3}{4}\left(1-5 \cos ^{2} i\right) \sin i \sin (f+\omega)$

$+\frac{5}{4} \sin ^{3}$ i $\sin (3 f+3 \omega)$

$S_{4}=+\frac{3}{4} \sin ^{2} i \cos 38 \cos (f+\omega)-\frac{3}{4} \sin ^{2} i \cos i \sin 3 \Omega \sin (f+\omega)$

$+\frac{1}{4}\left(1+3 \cos ^{2} i\right) \cos 3 \Omega \cos (3 f+3 \omega)-\frac{1}{4}\left(3+\cos ^{2} i\right) \cos$ is in $3 \Omega \sin (3 f+3 \omega)$,

$S_{5}=+\frac{3}{4} \sin ^{2}$ i s in $3 \delta \cos (f+\omega)+\frac{3}{4} \sin ^{2} i \cos i \cos 3 \varnothing s$ in $(f+\omega)$

$+\frac{1}{4}\left(1+3 \cos ^{2} i\right) \sin 3 \delta 6 \cos (3 f+3 \omega)+\frac{1}{4}\left(3+\cos ^{2} i\right) \cos i \cos 3 \delta \sin (3 f+3 \omega)$,

$S_{6}=-\frac{1}{4} \sin 2$ is in $28 \cos (f+\omega)+\frac{1}{4}\left(1-3 \cos ^{2} i\right) \sin i \cos 28 \sin (f+\omega)$

$(\cdot$

$+\frac{1}{4} \sin 2 i \sin 28 \cos (3 f+3 \omega)+\frac{1}{4}\left(1+\cos ^{2} i\right) \sin i \cos 2 \delta 6 \sin (3 f+3 \omega)$,

$S_{7}=+\frac{1}{8} \sin 2 i \cos 28 \cos (f+\omega)+\frac{1}{8}\left(1-3 \cos ^{2} i\right) \sin$ i s in $28 \sin (f+\omega)$

$-\frac{1}{8} \sin 2 i \cos 28 \cos (3 f+3 \omega)+\frac{1}{8}\left(1+\cos ^{2} i\right) \sin$ is in $28 \sin (3 f+3 \omega)$ 
Substituting these values of $S_{1}, S_{2}, \ldots, S_{7}$ into $v_{3}$ we obtain the following representation of the third part of the tidaldisturbing function $\Omega_{3}$ and of $R \cdot \nabla_{0} \Omega_{3}$ :

$$
\Omega_{3}=n^{2} a^{2} \cdot \frac{m^{\prime}}{M} \cdot a^{3} a^{\prime 3}\left(\frac{a^{\prime}}{r^{\prime}}\right)^{3}\left(\frac{a}{r}\right)^{4} .
$$

- $\left\{V_{31}^{(c)} \cos (f+\omega)+V_{31}^{(s)} \sin (f+\omega)+V_{33}^{(c)} \cos (3 f+3 \omega)+V_{33}^{(s)} \sin (3 f+3 \omega)\right\}$

$$
\begin{aligned}
& \text { R. } \nabla_{0} \Omega_{3}=n^{2} a^{2} \cdot \frac{m^{\prime}}{M} \cdot a^{3} a^{\prime 3}\left(\frac{a^{\prime}}{r^{\prime}}\right)^{3}\left(\frac{a}{r}\right)^{4} \\
& -\left\{V_{30}+V_{32}^{(c)} \cos (2 f+2 \omega)+V_{32}^{(s)} \sin (2 f+2 \omega)\right\},
\end{aligned}
$$

where

$$
\begin{aligned}
V_{31}^{(c)}= & -\frac{1}{4}\left(1-5 \cos ^{2} i\right) K_{31}^{(c)}+\frac{1}{4} K_{32}^{(c)} \sin 2 i+\frac{3}{4} K_{33}^{(c)} \sin ^{2} i \\
V_{31}^{(s)}= & -\frac{3}{4}\left(1-5 \cos ^{2}(i) K_{30}^{(s)} \sin i\right. \\
& -\frac{1}{4}\left(11-15 \cos ^{2} i\right) K_{31}^{(s)} \cos i+\frac{1}{4}\left(1-3 \cos ^{2} i\right) K_{32}^{(s)} \sin i, \\
& +\frac{3}{4} \sin ^{2} i \cos ^{(s-} K_{33}^{(s)} \\
V_{33}^{(c)}= & +\frac{5}{4} K_{31}^{(c)} \sin ^{2} i-\frac{1}{4} K_{32}^{(c)} \sin 2 i+\frac{1}{4}\left(1+3 \cos ^{2} i\right) K_{33}^{(c)}
\end{aligned}
$$




$$
\begin{aligned}
V_{33}^{(s)}= & +\frac{5}{4} K_{30}^{(s)} \sin ^{3} i+\frac{5}{4} K_{31}^{(s)} \sin ^{2} i \cos i+\frac{1}{4}\left(1+\cos ^{2} i\right) K_{32}^{(s)} \sin i \\
& +\frac{1}{4}\left(3+\cos ^{2} i\right) K_{33}^{(s)} \\
V_{30}= & -\frac{3}{2} \cos i \cdot\left(3-5 \cos ^{2} i\right) K_{30}^{(s)}+\frac{3}{2}\left(1-5 \cos ^{2} i\right) K_{31}^{(s)} \sin i \\
& +\frac{3}{2} \sin { }^{2} i \cos i \cdot K_{32}^{(s)}-\frac{3}{2} K_{33}^{(s)} \sin ^{3} i, \\
V_{32}^{(c)}= & +\frac{15}{2} K_{30}^{(s)} \sin { }^{2} i \cos i-\frac{5}{2}\left(1-3 \cos ^{2} i\right) K_{31}^{(s)} \sin i \\
V_{32}^{(s)}= & -\frac{5}{2} K_{31}^{(c)} \sin 2 i+K_{32}^{(c)} \cos 2 i+\frac{3}{2} K_{33}^{(c)} \sin 2 i \\
& -\frac{1}{2}\left(1-3 \cos ^{2} i\right) K_{32}^{(s)} \cos i-\frac{3}{2}\left(1+\cos ^{2} i\right) K_{33}^{(s)} \sin i
\end{aligned}
$$

and

$$
\begin{aligned}
& \mathrm{K}_{31}^{(\mathrm{c})}=+\mathrm{S}_{1}^{\prime} \cos \delta+\mathrm{S}_{2}^{\prime} \sin \delta, \\
& \mathrm{K}_{32}^{(\mathrm{c})}=-\mathrm{S}_{6}^{\prime} \sin 2 \delta+\frac{1}{2} \mathrm{~S}_{7}^{\prime} \cos 2 \delta, \\
& \mathrm{K}_{33}^{(\mathrm{c})}=+\mathrm{S}_{4}^{\prime} \cos 3 \delta+\mathrm{S}_{5}^{\prime} \sin 3 \delta,
\end{aligned}
$$




$$
\begin{aligned}
& \mathrm{K}_{30}^{(s)}=\mathrm{S}_{3}^{\prime}, \\
& \mathrm{K}_{31}^{(s)}=-\mathrm{S}_{1}^{\prime} \sin \delta+\mathrm{S}_{2}^{\prime} \cos \delta, \\
& \mathrm{K}_{32}^{(\mathrm{s})}=+\mathrm{S}_{6}^{\prime} \cos 2 \delta+\frac{1}{2} \mathrm{~S}_{7}^{\prime} \sin 2 \delta, \\
& \mathrm{K}_{33}^{(\mathrm{s})}=-\mathrm{S}_{4}^{\prime} \sin 3 \delta+\mathrm{S}_{5}^{\prime} \cos 3 \delta .
\end{aligned}
$$

In a similar manner we deduce:

$$
\begin{aligned}
V_{4}=V_{40} & +V_{42}^{(c)} \cos (2 f+2 \omega)+V_{42}^{(s)} \sin (2 f+2 \omega) \\
& +V_{44}^{(c)} \cos (4 f+4 \omega)+V_{44}^{(s)} \sin (4 f+4 \omega)
\end{aligned}
$$

where

$$
\begin{aligned}
\mathrm{V}_{40}= & +\frac{3}{8}\left(3-30 \cos ^{2} \mathrm{i}+35 \cos ^{4} \mathrm{i}\right) \mathrm{K}_{40}^{(c)} \\
+ & \frac{1}{16} \sin 2 \mathrm{i} \cdot\left(3-7 \cos ^{2} \mathrm{i}\right) \mathrm{K}_{41}^{(\mathrm{c})}-\frac{3}{8} \sin ^{2} \mathrm{i}\left(1-7 \cos ^{2} \mathrm{i}\right) \mathrm{K}_{42}^{(\mathrm{c})} \\
-\frac{3}{8} \sin ^{3} \mathrm{i} \cos \mathrm{i} \cdot \mathrm{K}_{43}^{(\mathrm{c})}, & \\
\mathrm{V}_{42}^{(c)}= & -\frac{5}{2} \sin ^{2} \mathrm{i}\left(1-7 \cos ^{2} \mathrm{i}\right) \mathrm{K}_{40}^{(c)} \\
& -\frac{1}{12} \sin ^{(\mathrm{i}} \mathrm{i} \cdot\left(4-7 \cos ^{2} \mathrm{i}\right) \mathrm{K}_{41}^{(\mathrm{c})} \\
& +\frac{1}{2}\left(1-6 \cos ^{2} \mathrm{i}+7 \cos ^{4} \mathrm{i}\right) \mathrm{K}_{42}^{(c)} \\
& -\frac{1}{2} \cos ^{3} \mathrm{i} \sin ^{\mathrm{i}} \cdot \mathrm{K}_{43}^{(c)},
\end{aligned}
$$




$$
\begin{aligned}
& V_{42}^{(s)}=-\frac{1}{12}\left(1-7 \cos ^{2} i\right) \sin i \cdot K_{41}^{(s)} \\
& +\frac{1}{2}\left(5-7 \cos ^{2} i\right) \cos i \cdot K_{42}^{(s)} \\
& +\frac{1}{4}\left(1-3 \cos ^{2} i\right) \sin i \cdot K_{43}^{(s)} \\
& V_{44}^{(c)}=+\frac{35}{8} \sin ^{4} \mathrm{i} \cdot \mathrm{K}_{40}^{(\mathrm{c})}+\frac{7}{24} \sin ^{3} \mathrm{i} \cos \mathrm{i} \cdot \mathrm{K}_{41}^{(\mathrm{c})} \\
& +\frac{7}{8}\left(1+\cos ^{2} i\right) \sin ^{2} i \cdot K_{42}^{(c)}+\frac{1}{16}\left(3+\cos ^{2} i\right) \cdot \sin 2 i \cdot K_{43}^{\left(c^{*}\right)}, \\
& V_{44}^{(s)}=+\frac{7}{24} \sin ^{3} i \cdot K_{41}^{(s)}-\frac{7}{4} \sin ^{2} i \cos i \cdot K_{42}^{(s)} \\
& +\frac{1}{8}\left(1+3 \cos ^{2} i\right) \sin i \cdot K_{43}^{(s)} \\
& R \cdot \nabla_{0} V_{4}=V_{41}^{(c)} \cos (f+\omega)+V_{41}^{(s)} \sin (f+\omega) \\
& +V_{43}^{(c)} \cos (3 f+3 \omega)+V_{43}^{(s)} \sin (3 f+3 \omega)
\end{aligned}
$$

where

$$
\begin{aligned}
V_{41}^{(c)}= & -\frac{1}{4}\left(3-7 \cos ^{2} i\right) \cos i \cdot K_{41}^{(s)}-\frac{3}{2} \sin i\left(1-7 \cos ^{2} i\right) \cdot K_{42}^{(s)} \\
& +\frac{9}{4} \sin ^{2} i \cos i \cdot K_{43}^{(s)}
\end{aligned}
$$




$$
\begin{aligned}
V_{41}^{(s)}= & +15 \sin i \cos i\left(3-7 \cos ^{2} i\right) \cdot K_{40}^{(c)} \\
& -\frac{1}{4}\left(3-27 \cos ^{2} i+28 \cos ^{4} i\right) \cdot K_{41}^{(c)} \\
& -3 \sin i \cos i\left(4-7 \cos ^{2} i\right) \cdot K_{42}^{(c)} \\
& +\frac{3}{4} \sin ^{2} i\left(1-4 \cos ^{2} i\right) \cdot K_{43}^{(c)} \\
V_{43}^{(c)}= & +\frac{7}{4} \sin ^{2} i \cos i \cdot K_{41}^{(s)}+\frac{7}{2} \sin i \cdot\left(1-3 \cos ^{2} i\right) \cdot K_{42}^{(s)} \\
& -\frac{1}{4}\left(5-9 \cos ^{2} i\right) \cos i \cdot K_{43}^{(s)} \\
V_{43}^{(s)}= & -35 \sin ^{3} i \cos ^{(s} \cdot K_{40}^{(c)}+\frac{7}{12} \sin ^{2} i\left(1-4 \cos ^{2} i\right) \cdot K_{41}^{(c)} \\
& -7 \sin ^{(c)} \cos ^{3} i \cdot K_{42}^{(c)}+\frac{1}{4}\left(3-3 \cos ^{2} i-4 \cos ^{4} i\right) \cdot K_{43}^{(c)}
\end{aligned}
$$

and

$$
\begin{aligned}
& \mathrm{K}_{40}^{(\mathrm{c})}=+\mathrm{T}_{3}^{\prime}, \\
& \mathrm{K}_{41}^{(\mathrm{c})}=+\mathrm{T}_{1}^{\prime} \sin \delta-\mathrm{T}_{2}^{\prime} \cos \delta \\
& \mathrm{K}_{42}^{(\mathrm{c})}=+\mathrm{T}_{6}^{\prime} \cos 2 \delta+\frac{1}{2} \mathrm{~T}_{7}^{\prime} \sin 2 \delta \\
& \mathrm{K}_{43}^{(\mathrm{c})}=+\mathrm{T}_{4}^{\prime} \sin 3 \delta_{6}-\mathrm{T}_{5}^{\prime} \cos 3 \delta_{6}
\end{aligned}
$$




$$
\begin{gathered}
\mathrm{K}_{41}^{(\mathrm{s})}=+\mathrm{T}_{1}^{\prime} \cos \delta+\mathrm{T}_{2}^{\prime} \sin \Omega, \\
\because \\
\mathrm{K}_{42}^{(\mathrm{s})}=+\mathrm{T}_{6}^{\prime} \sin 2 \delta-\frac{1}{2} \mathrm{~T}_{7}^{\prime} \cos 2 \delta, \\
\mathrm{K}_{43}^{(\mathrm{s})}=+\mathrm{T}_{4}^{\prime} \cos 3 \delta+\mathrm{T}_{5}^{\prime} \sin 3 \delta .
\end{gathered}
$$

\section{DIFFERENTIAL EQUATIONS FOR VARIATION OF CONSTANTS}

Only the long period tidal effects in the elements can be easily observed. To determine them, we make use of the classical equations for variation of elements with the short period terms eliminated by averaging the right hand sides over the instantaneous orbit of the satellite:

$$
\begin{gathered}
\frac{d e}{d t}=-\frac{\sqrt{1-e^{2}}}{n a^{2} e} \cdot \frac{1}{2 \pi} \int_{0}^{2 \pi} \frac{\partial \Omega}{\partial f} d g, \\
\frac{d \pi}{d t}=+\frac{\sqrt{1-e^{2}}}{n a^{2} e} \cdot \frac{1}{2 \pi} \int_{0}^{2 \pi}\left\{-a \frac{\partial \Omega}{\partial r} \cos f+\left(\frac{a}{r}+\frac{1}{1-e^{2}}\right) \frac{\partial \Omega}{\partial f} \sin f\right\} d g \\
+2 \sin ^{2} \frac{i}{2} \frac{d \Omega}{d t}, \\
\sin i \frac{d \Omega}{d t}=+\frac{1}{n a^{2} \sqrt{1-e^{2}}} \cdot \frac{1}{2 \pi} \int_{0}^{2 \pi}\left(r \frac{\partial \Omega}{\partial z}\right) \sin (f+\omega) d g,
\end{gathered}
$$




$$
\begin{array}{r}
\frac{d i}{d t}=+\frac{1}{n a^{2} \sqrt{1-e^{2}}} \cdot \frac{1}{2 \pi} \int_{0}^{2 \pi}\left(r \frac{\partial \Omega}{\partial Z}\right) \cos (f+\omega) d g . \\
\frac{d L}{d t}=-\frac{2}{n a^{2}} \cdot \frac{1}{2 \pi} \int_{0}^{2 \pi} r \frac{\partial \Omega}{\partial r} d g+\left(1-\sqrt{\left.1-e^{2}\right)} \frac{d \pi}{d t}+2 \sqrt{1-e^{2}} \sin ^{2} \frac{i}{2} \frac{d}{d t} .\right.
\end{array}
$$

In our case the disturbing force can be conveniently represented in the form:

$$
\nabla \Omega=\frac{\partial \Omega}{\partial \mathbf{r}} \mathbf{r}^{0}+\nabla \mathbf{r}^{0} \cdot \nabla_{0} \Omega
$$

$\nabla_{0} \Omega$ is the gradient of $\Omega$ with respect to $\mathbf{r}^{0}$ and

$$
\nabla \mathbf{r}^{0}=\frac{1}{r}\left(I-\mathbf{r}^{0} \mathbf{r}^{0}\right)
$$

From (44) and (45) we deduce:

$$
\mathbf{r} \frac{\partial \Omega}{\partial \mathbf{Z}}=\mathbf{r} \mathbf{R} \cdot \nabla \Omega=\mathbf{R} \cdot \nabla_{0} \Omega
$$

Substituting (47) into (42) and (43) and taking

$$
d g=\frac{r^{2}}{a^{2}} \cdot \frac{d f}{\sqrt{1-e^{2}}}
$$

into account, we deduce:

$$
\frac{\mathrm{de}}{\mathrm{dt}}=-\frac{1}{n a^{2} \mathrm{e}} \cdot \frac{1}{2 \pi} \int_{0}^{2 \pi}\left(\frac{\mathrm{r}}{\mathrm{a}}\right)^{2} \frac{\partial \Omega}{\partial \mathrm{f}} \mathrm{df}
$$




$$
\begin{aligned}
\frac{\mathrm{d} \pi}{\mathrm{dt}}=+\frac{1}{n \mathrm{a}^{2} \mathrm{e}} \cdot \frac{1}{2 \pi} \int_{0}^{2 \pi}\left(\frac{r}{\mathrm{a}}\right)^{2}\{ & \left.-\mathrm{a} \frac{\partial \Omega}{\partial \mathrm{r}} \cos \mathrm{f}+\left(\frac{\mathrm{a}}{\mathrm{r}}+\frac{1}{1-\mathrm{e}^{2}}\right) \frac{\partial \Omega}{\partial \mathrm{f}} \sin \mathrm{f}\right\} \mathrm{df} \\
& +2 \sin ^{2} \frac{\mathrm{i}}{2} \frac{\mathrm{d} \Omega}{\mathrm{dt}}
\end{aligned}
$$

$$
\begin{aligned}
& \sin i \frac{d \delta}{d t}=+\frac{1}{n a^{2}\left(1-e^{2}\right)} \cdot \frac{1}{2 \pi} \int_{0}^{2 \pi}\left(\frac{r}{a}\right)^{2}\left(\mathbf{R} \cdot \nabla_{0} \Omega\right) \sin (f+\omega) d f, \\
& \frac{\mathrm{di}}{\mathrm{dt}}=+\frac{1}{n \mathrm{a}^{2}\left(1-\mathrm{e}^{2}\right)} \cdot \frac{1}{2 \pi} \cdot \int_{0}^{2 \pi}\left(\frac{\mathrm{r}}{\mathrm{a}}\right)^{2}\left(\mathbf{R} \cdot \nabla_{0} \Omega\right) \cos (\mathrm{f}+\omega) \mathrm{df} \\
& \frac{\mathrm{dL}}{\mathrm{dt}}=-\frac{2}{n \mathrm{a}^{2} \sqrt{1-\mathrm{e}^{2}}} \cdot \frac{1}{2 \pi} \int_{0}^{2 \pi} \cdot\left(\frac{\mathrm{r}}{\mathrm{a}}\right)^{2} \cdot\left(\mathrm{r} \frac{\partial \Omega}{\partial \mathrm{r}}\right) \mathrm{df} \\
& +\left(1-\sqrt{1-\mathrm{e}^{2}}\right) \frac{\mathrm{d} \pi}{\mathrm{dt}}+2 \sqrt{1-\mathrm{e}^{2}} \sin ^{2} \frac{\mathrm{i}}{2} \frac{\mathrm{d} \delta}{\mathrm{dt}} .
\end{aligned}
$$

In the main problem we have:

$$
\Omega_{2}=n^{2} a^{2} \frac{m^{\prime}}{M} a^{2} a^{\prime 3}\left(\frac{a^{\prime}}{r^{\prime}}\right)^{3}\left(\frac{a}{r}\right)^{3}\left[V_{20}+V_{22}^{(c)} \cos (2 f+2 \omega)+V_{22}^{(s)} \sin (2 f+2 \omega)\right]
$$

and

$$
\mathbf{R} \cdot \ddot{\nabla}_{0} \Omega_{2}=n^{2} a^{2} \frac{m^{\prime}}{M} a^{2} \alpha^{\prime 3}\left(\frac{a^{\prime}}{r^{\prime}}\right)^{3}\left(\frac{a}{r}\right)^{3}\left[v_{21}^{(c)} \cos (f+\omega)+v_{21}^{(s)} \sin (f+\omega)\right]
$$


where $V_{20}, V_{22}^{(c)}, V_{22}^{(s)}$ are given by the equations $(11)-(13)$ and $V_{21}^{(c)}, V_{21}^{(s)}$ by (14) - (15).

Substituting (53) and (54) into (48) - (52) we obtain, after easy transformations, the following expressions for the perturbations as caused by $\Omega_{2}$ :

$$
\begin{aligned}
& \frac{\mathrm{d} \delta_{2} \mathrm{e}}{\mathrm{dt}}=0 \\
& \frac{d \delta_{2} \pi}{d t}=+\frac{3 n a^{2} a^{\prime 3}}{\left(1-e^{2}\right)^{2}} \cdot \frac{m^{\prime}}{M} \cdot\left(\frac{a^{\prime}}{r^{\prime}}\right)^{3} v_{20}+2 \sin ^{2} \frac{i}{2} \frac{d \delta_{2} \delta}{d t} \\
& \sin i \frac{d \delta_{2} \delta}{d t}=+\frac{n a^{2} \alpha^{\prime 3}}{2\left(1-e^{2}\right)^{2}} \cdot \frac{m^{\prime}}{M} \cdot\left(\frac{a^{\prime}}{r^{\prime}}\right)^{3} V_{21}^{(s)} \\
& \frac{d \delta_{2} i}{d t}=+\frac{n a^{2} a^{\prime 3}}{2\left(1-e^{2}\right)^{2}} \cdot \frac{m^{\prime}}{M} \cdot\left(\frac{a^{\prime}}{r^{\prime}}\right)^{3} V_{21}^{(c)} \\
& \frac{d \delta_{2} L}{d t}=+\frac{6 n a^{2} a^{\prime 3}}{\left(1-e^{2}\right)^{3 / 2}} \cdot \frac{m^{\prime}}{M} \cdot\left(\frac{a^{\prime}}{r^{\prime}}\right)^{3} v_{20}+\frac{e}{1+\sqrt{1-e^{2}}} \frac{d \delta_{2} \pi}{d t}+2 \sqrt{1-e^{2}} \sin ^{2} \frac{i}{2} \frac{d \delta_{2} \delta}{d t} .
\end{aligned}
$$

In limits of the "main problem" the eccentricity of the artificial satellite is not affected by the tidal perturbations. This result was obtained by Kozai (1965) using the differential equation for the general perturbations in satellite's eccentricity.

For the perturbative effects caused by $\Omega_{3}$ we obtain, making use of (16) - (17):

$$
\frac{\mathrm{d} \tilde{\delta}_{3} e}{\mathrm{dt}}=+\frac{\mathrm{n} a^{3} a^{\prime 3}}{\left(1-\mathrm{e}^{2}\right)^{2}} \cdot \frac{\mathrm{m}^{\prime}}{M} \cdot\left(\frac{\mathrm{a}^{\prime}}{\mathrm{r}^{\prime}}\right)^{3} \cdot\left(\mathrm{V}_{31}^{(\mathrm{c})} \sin \omega-\mathrm{V}_{31}^{(\mathrm{s})} \cos \omega\right)
$$




$$
\frac{\mathrm{d} \delta_{3} \pi}{\mathrm{dt}}=\frac{\mathrm{n} \alpha^{3} a^{\prime 3}}{\left(1-\mathrm{e}^{2}\right)^{3} \mathrm{e}} \cdot \frac{\mathrm{m}^{\prime}}{\mathrm{M}} \cdot\left(\frac{\mathrm{a}^{\prime}}{\mathrm{r}^{\prime}}\right)^{-3} \cdot\left(1+4 \mathrm{e}^{2}\right)\left(\mathrm{V}_{31}^{(\mathrm{c})} \cos \omega+\mathrm{V}_{31}^{(\mathrm{s})} \sin \omega\right)
$$

$$
+2 \sin ^{2} \frac{i}{2} \frac{d \delta_{3} \delta}{d t}
$$

$\sin i \frac{\mathrm{d} \delta_{3} \delta}{\mathrm{dt}}=\frac{\mathrm{n} \alpha^{3} a^{\prime 3} \mathrm{e}}{\left(1-\mathrm{e}^{2}\right)^{3}} \cdot \frac{\mathrm{m}^{\prime}}{\mathrm{M}} \cdot\left(\frac{\mathrm{a}^{\prime}}{\mathrm{r}^{\prime}}\right)^{3} \cdot\left(\mathrm{v}_{30} \sin \omega-\frac{1}{2} \mathrm{~V}_{32}^{(\mathrm{c})} \sin \omega+\frac{1}{2} \mathrm{~V}_{32}^{(\mathrm{s})} \cos \omega\right)$

$$
\begin{gathered}
\frac{\mathrm{d} \delta_{3} \mathrm{i}}{\mathrm{dt}}=\frac{\mathrm{n} \alpha^{3} \alpha^{\prime 3} \mathrm{e}}{\left(1-\mathrm{e}^{2}\right)^{3}} \cdot \frac{\mathrm{m}^{\prime}}{\mathrm{M}} \cdot\left(\frac{\mathrm{a}^{\prime}}{\mathrm{r}^{\prime}}\right)^{3} \cdot\left(\mathrm{V}_{30} \cos \omega+\frac{1}{2} \mathrm{~V}_{32}^{(\mathrm{c})} \cos \omega+\frac{1}{2} \mathrm{~V}_{32}^{(\mathrm{s})} \sin \omega\right) \\
\frac{\mathrm{d} \delta_{3} \mathrm{~L}}{\mathrm{dt}}=+\frac{8 n \alpha^{3} \alpha^{\prime 3} \mathrm{e}}{\left(1-\mathrm{e}^{2}\right)^{5 / 2}} \cdot \frac{\mathrm{m}^{\prime}}{\mathrm{M}} \cdot\left(\frac{\mathrm{a}^{\prime}}{\mathrm{r}^{\prime}}\right)^{3} \cdot\left(\mathrm{V}_{31}^{(\mathrm{c})} \cos \omega+\mathrm{V}_{31}^{(\mathrm{s})} \sin \omega\right) \\
\quad+\frac{\mathrm{e}}{1+\sqrt{1-\mathrm{e}^{2}}} \frac{\mathrm{d} \delta_{3} \pi}{\mathrm{dt}}+2 \sqrt{1-\mathrm{e}^{2}} \sin ^{2} \frac{\mathrm{i}}{2} \frac{\mathrm{d} \delta_{3} \delta}{\mathrm{dt}}
\end{gathered}
$$

Similarly, for the effects caused by the part $\Omega_{4}$ of the disturbing function we have, making use of (27) and (33):

$$
\begin{aligned}
& \frac{d \delta_{4} e}{d t}=+\frac{3 n a^{4} a^{\prime 3} e}{2\left(1-e^{2}\right)^{3}} \cdot \frac{m^{\prime}}{M} \cdot\left(\frac{a^{\prime}}{r^{\prime}}\right)^{3}\left(+V_{42}^{(c)} \sin 2 \omega-V_{42}^{(s)} \cos 2 \omega\right) \\
\frac{d \delta_{4} \pi}{d t}= & +\frac{n \alpha^{4} a^{\prime 3}}{\left(1-e^{2}\right)^{4}} \cdot \frac{m^{\prime}}{M} \cdot\left(\frac{a^{\prime}}{r^{\prime}}\right)^{3}\left\{+\left(10+\frac{15}{2} e^{2}\right) V_{40}\right. \\
& \left.+\left(\frac{3}{2}+\frac{15}{4} e^{2}\right)\left(+V_{42}^{(c)} \cos 2 \omega+V_{42}^{(s)} \sin 2 \omega\right)\right\}+2 \sin ^{2} \frac{i}{2} \frac{d \delta_{4} \delta}{d t},
\end{aligned}
$$




$$
\begin{aligned}
\sin i \frac{d \delta_{4} \delta}{d t}= & +\frac{n a^{4} a^{\prime 3}}{2\left(1-e^{2}\right)^{4}} \cdot \frac{m^{\prime}}{M} \cdot\left(\frac{a^{\prime}}{r^{\prime}}\right)^{3}\left\{+\left(1+\frac{3}{2} e^{2}\right) V_{41}^{(s)}\right. \\
& \left.+\frac{3}{4} e^{2}\left[+\left(-V_{41}^{(s)}+V_{43}^{(s)}\right) \cos 2 \omega+\left(+V_{41}^{(c)}-V_{43}^{(c)}\right) \sin 2 \omega\right]\right\} \\
\frac{d \delta_{4} i}{d t}= & +\frac{n a^{4} a^{\prime 3}}{2\left(1-e^{2}\right)^{4}} \cdot \frac{m^{\prime}}{M} \cdot\left(\frac{a^{\prime}}{r^{\prime}}\right)^{3}\left\{\left(1+\frac{3}{2} e^{2}\right) V_{41}^{(c)}\right. \\
& \left.+\frac{3}{4} e^{2}\left[+\left(+V_{41}^{(c)}+V_{43}^{(c)}\right) \cos 2 \omega+\left(V_{41}^{(s)}+V_{43}^{(s)}\right) \sin 2 \omega\right]\right\} \\
\frac{d \delta_{4} L}{d t}= & +\frac{10 n a^{4} a^{\prime 3}}{\left(1-e^{2}\right)^{7 / 2}} \cdot \frac{m^{\prime}}{M} \cdot\left(\frac{a^{\prime}}{r^{\prime}}\right)^{3} \cdot\left\{\left(1+\frac{3}{2} e^{2}\right) V_{40}+\right. \\
& \left.+\frac{3}{4} e^{2}\left(V_{42}^{(c)} \cos 2 \omega+V_{42}^{(s)} \sin 2 \omega\right)\right\}+\frac{e}{1+\sqrt{1-e^{2}}} \frac{d t}{d t}+2 \sqrt{1-e^{2}} s^{2} n^{2} \frac{i}{2} \frac{d \delta_{4} \delta}{d t}
\end{aligned}
$$

\section{CONCLUSION}

The present theory of the tidal effects represents an improvement over the theory based on constancy of Love numbers. The differential equations for the tidal effects in the elements of an artificial satellite are given in the present article in a form convenient for numerical integration over a long interval of time. This form is also suitable for representing the tidal effects as trigonometric series with the arguments $\omega, \delta$ of the satellite and $\ell, \ell^{\prime}, F, D, \Gamma$ of the Moon. The numerical integration can be performed using some simple quadrature formula, without resorting to a predictor-corrector system. The final goal is the determination of the Love numbers $k_{2}, k_{3}, \ldots$ which we assumed to be not constant, but latitude dependent. This assumption required the reformulation of the "main problem" of the theory of tidal effects. 
In the present exposition, the main problem contains the influence of oblateness and the deviation of $k_{2}$ and $k_{3}$ from constants. The effects caused by $k_{2}$ and $k_{3}$ (including their deviations from mean values) are the most significant ones. It is extremely doubtful that the effects caused by $k_{4}, k_{5}, \ldots$ are significant enough to be detected.

The computation done in the previous work (Musen and Estes, 1971) shows that the tidal perturbations in satellite orbital elements can be a few seconds of arc. The deviation of $k_{2}$ and $k_{3}$ from constants will probably contribute effects on the order of $0 ! 1-1 ! \cdot 0$.

Some guess concerning a future direction of work can be made. We might expect that with the accumulation of observational material the problem of separation of the long period tidal effects caused by the solid Earth from the effects caused by oceanic tides, as well as the problem of the non-homogeneous structure of the Earth, will arise.

\section{COLLECTION OF FORMULAS}

Basic information:

Given:

$1^{\circ}$ the mean elements of the satellite

$$
\text { a, } \omega_{0}, \delta_{0}, e, i \text {, and the mean mean motion } \mathrm{n}_{0} \text {, }
$$

$\omega_{0}$ and $\delta_{0}$ are referred to the mean equator and equinox of a given initial epoch

$t_{0}$

$2^{\circ} \quad$ Lunar coordinates

$$
x^{\prime}, y^{\prime}, z^{\prime}
$$

referred to the same equator and equinox. 
$3^{\circ}$ We have (Brouwer, 1959):

$$
\begin{aligned}
& \omega=\omega_{0}+n_{0} \omega_{1}\left(t-t_{0}\right) \\
& \delta_{6}=\delta_{0}+n_{0} \delta_{1}\left(t-t_{0}\right)
\end{aligned}
$$

where

$$
\begin{aligned}
\omega_{1}= & +\frac{3}{2} \gamma_{2}^{\prime}\left(-1+5 \theta^{2}\right)+\frac{3}{32} \gamma_{2}^{\prime 2}\left[\left(-35+24 \eta+25 \eta^{2}\right)\right. \\
& \left.+\left(90-192 \eta-126 \eta^{2}\right) \theta^{2}+\left(385+360 \eta+45 \eta^{2}\right) \theta^{4}\right] \\
& +\frac{5}{16} \gamma_{4}^{\prime}\left[\left(21-9 \eta^{2}\right)+\left(-270+126 \eta^{2}\right) \theta^{2}+\left(385-189 \eta^{2}\right) \theta^{4}\right], \\
\delta_{1}= & -3 \gamma_{2}^{\prime} \theta+\frac{3}{8} \gamma_{2}^{\prime 2}\left[\left(-5+12 \eta+9 \eta^{2}\right) \theta+\left(-35-36 \eta-5 \eta^{2}\right) \theta^{2}\right] \\
& \left.+\frac{5}{4} \gamma_{4}^{\prime}\left(5-3 \eta^{2}\right)\left(3 \theta-7 \theta^{3}\right)\right] .
\end{aligned}
$$

and

$$
\gamma_{2}^{\prime}=+\frac{1}{2} \cdot \frac{\mathrm{J}_{2} \mathrm{R}^{2}}{\mathbf{a}^{2}} \cdot \eta^{-4}, \gamma_{4}^{\prime}=-\frac{3}{8} \cdot \frac{\mathrm{J}_{4} \mathrm{R}^{2}}{\mathrm{a}^{4}} \cdot \eta^{-8}, \quad \eta=\sqrt{1-\mathrm{e}^{2}}, \quad \theta=\cos \mathrm{i}
$$

$4^{\circ}$

$$
\begin{aligned}
& r^{\prime}=\sqrt{x^{\prime 2}+y^{\prime 2}+z^{\prime 2}} \\
& \lambda^{\prime}=x^{\prime} / r^{\prime}, \quad \mu^{\prime}=y^{\prime} / r^{\prime}, \quad \nu^{\prime}=z^{\prime} / r^{\prime}
\end{aligned}
$$

$5^{\circ}$

$$
\begin{array}{ll}
\mathrm{D}_{0}^{\prime}=1-3 \nu^{\prime 2} & \mathrm{E}_{0}^{\prime}=\frac{\mathrm{a}^{\prime}}{\mathrm{r}^{\prime}} \nu^{\prime}\left(3-5 \nu^{\prime 2}\right) \\
\mathrm{D}_{1}^{\prime}=\lambda^{\prime 2}-\mu^{\prime 2} & \mathrm{E}_{1}^{\prime}=\frac{\mathrm{a}^{\prime}}{\mathrm{r}^{\prime}} \lambda^{\prime}\left(1-5 \nu^{\prime 2}\right)
\end{array}
$$




$$
\begin{aligned}
& \mathrm{D}_{2}^{\prime}=\lambda^{\prime} \mu^{\prime} \quad \mathrm{E}_{2}^{\prime}=\frac{\mathbf{a}^{\prime}}{\mathrm{r}^{\prime}} \mu^{\prime}\left(1-\dot{5} \nu^{\prime 2}\right) \\
& \mathrm{D}_{3}^{\prime}=\lambda^{\prime} \nu^{\prime} \quad \mathrm{E}_{3}^{\prime}=\frac{\mathrm{a}^{\prime}}{\mathrm{r}^{\prime}} \nu^{\prime}\left(\lambda^{\prime 2}-\mu^{\prime 2}\right) \\
& \mathrm{D}_{4}^{\prime}=\mu^{\prime} \nu^{\prime} \quad \mathrm{E}_{4}^{\prime}=\frac{\mathbf{a}^{\prime}}{\mathbf{r}^{\prime}} \lambda^{\prime} \mu^{\prime} \nu^{\prime} \\
& E_{5}^{\prime}=\frac{a^{\prime}}{r^{\prime}} \lambda^{\prime}\left(\lambda^{\prime 2}-3 \mu^{\prime 2}\right) \\
& E_{6}^{\prime}=\frac{a^{\prime}}{r^{\prime}} \mu^{\prime}\left(3 \lambda^{\prime 2}-\mu^{\prime 2}\right)
\end{aligned}
$$

Main problem: perturbations produced by $\Omega_{2}$.

$$
\begin{aligned}
& a_{20}=+\frac{1}{4}\left(k_{20}+\frac{2}{7} k_{22}\right)\left(1-\frac{55}{42} \epsilon^{2}\right) \\
& \beta_{20}=+\frac{3}{28} h_{31} \cdot a^{\prime} \\
& a_{21}=+\frac{3}{4}\left(k_{20}-\frac{2}{7} k_{22}\right)\left(1-\frac{5}{14} \epsilon^{2}\right) \\
& \beta_{21}=+\frac{15}{28} \mathrm{k}_{31} a^{\prime} \\
& a_{22}=+3\left(k_{20}-\frac{2}{7} k_{22}\right)\left(1-\frac{5}{14} \epsilon^{2}\right) \\
& \beta_{22}=+\frac{15}{7} \mathrm{k}_{31} a^{\prime} \\
& a_{23}=a_{24}=+3\left(k_{20}+\frac{1}{7} k_{22}\right)\left(1-\frac{15}{14} \epsilon^{2}\right) \\
& \beta_{23}=\beta_{24}=-\frac{3}{7} k_{31} a^{\prime} \\
& c_{200}=-\frac{1}{2}+\frac{3}{2} \cos ^{2} \mathrm{i} \quad c_{211}=+\cos \mathrm{i} \quad \mathrm{s}_{210}=-3 \sin 2 \mathrm{i} \\
& c_{201}=-\frac{1}{4} \sin 2 \mathrm{i} \quad \mathrm{c}_{212}=+2 \sin \mathrm{i} \quad \mathrm{s}_{211}=-\cos 2 \mathrm{i} \\
& c_{202}=+\frac{1}{2} \sin ^{2} i \\
& \mathrm{~s}_{212}=+\sin 2 \mathrm{i}
\end{aligned}
$$




$$
\begin{array}{ll}
\mathrm{P}_{0}^{\prime}=+a_{20} \mathrm{D}_{0}^{\prime}+\beta_{20} \mathrm{E}_{0}^{\prime} & \mathrm{K}_{20}^{(\mathrm{c})}=+\mathrm{P}_{0} \\
\mathrm{P}_{1}^{\prime}=+\alpha_{21} \mathrm{D}_{1}^{\prime}+\beta_{21} \mathrm{E}_{3}^{\prime} & \mathrm{K}_{21}^{(\mathrm{c})}=+\mathrm{P}_{3}^{\prime} \sin \Omega-\mathrm{P}_{4}^{\prime} \cos \delta \\
\mathrm{P}_{2}^{\prime}=+a_{22} \mathrm{D}_{2}^{\prime}+\beta_{22} \mathrm{E}_{4}^{\prime} & \mathrm{K}_{22}^{(\mathrm{c})}=+\mathrm{P}_{1}^{\prime} \cos 2 \delta+\frac{1}{2} \mathrm{P}_{2}^{\prime} \sin 2 \delta b \\
\mathrm{P}_{3}^{\prime}=+a_{23} \mathrm{D}_{3}^{\prime}+\beta_{23} \mathrm{E}_{1}^{\prime} & \mathrm{K}_{21}^{(\mathrm{s})}=+\mathrm{P}_{3}^{\prime} \cos \delta+\mathrm{P}_{4}^{\prime} \mathrm{sin} \delta \\
\mathrm{P}_{4}^{\prime}=+a_{24} \mathrm{D}_{4}^{\prime}+\beta_{24} \mathrm{E}_{2}^{\prime} & \mathrm{K}_{22}^{(\mathrm{s})}=+\mathrm{P}_{1}^{\prime} \mathrm{sin} 2 \delta-\frac{1}{2} \mathrm{P}_{2}^{\prime} \cos 2 \delta \\
\mathrm{V}_{20}=+\mathrm{c}_{200} \mathrm{~K}_{20}^{(\mathrm{c})}+\mathrm{c}_{201} \mathrm{~K}_{21}^{(\mathrm{c})}+\mathrm{c}_{202} \mathrm{~K}_{22}^{(\mathrm{c})} \\
\mathrm{V}_{21}^{(\mathrm{s})}=+\mathrm{s}_{210} \mathrm{~K}_{20}^{(\mathrm{c})}+\mathrm{s}_{211} \mathrm{~K}_{21}^{(\mathrm{c})}+\mathrm{s}_{212} \mathrm{~K}_{22}^{(\mathrm{c})} \\
\mathrm{V}_{21}^{(\mathrm{c})}=
\end{array}
$$

$\sin i \frac{d \delta_{2} \delta}{d t}=+\frac{n a^{2} a^{\prime 3}}{2\left(1-e^{2}\right)^{2}} \cdot \frac{m^{\prime}}{M} \cdot\left(\frac{a^{\prime}}{r^{\prime}}\right)^{3} V_{21}^{(s)}$

$$
\frac{\mathrm{d} \delta_{2} \mathrm{i}}{\mathrm{dt}}=+\frac{\mathrm{n} \alpha^{2} \alpha^{\prime 3}}{2\left(1-\mathrm{e}^{2}\right)^{2}} \cdot \frac{\mathrm{m}^{\prime}}{M} \cdot\left(\frac{\mathrm{a}^{\prime}}{\mathrm{r}^{\prime}}\right)^{3} \mathrm{~V}_{21}^{(\mathrm{c})}
$$$$
\frac{\mathrm{d} \delta_{2} \pi}{\mathrm{dt}}=+\frac{3 \mathrm{n} \alpha^{2} \alpha^{\prime 3}}{\left(1-\mathrm{e}^{2}\right)^{2}} \cdot \frac{\mathrm{m}^{\prime}}{\mathrm{M}} \cdot\left(\frac{\mathrm{a}^{\prime}}{\mathrm{r}^{\prime}}\right)^{3} \mathrm{~V}_{20}+2 \sin ^{2} \frac{\mathrm{i}}{2} \frac{\mathrm{d} \delta_{2} \delta}{\mathrm{dt}}
$$$$
\frac{\mathrm{d} \delta_{2} \mathrm{e}}{\mathrm{dt}}=0
$$$$
\frac{d \delta_{2} L}{d t}=+\frac{6 n a^{2} a^{\prime 3}}{\left(1-e^{2}\right)^{3 / 2}} \cdot \frac{m^{\prime}}{M} \cdot\left(\frac{a^{\prime}}{r^{\prime}}\right)^{3} \cdot V_{20}+\frac{e}{1+\sqrt{1-e^{2}}} \cdot \frac{d \delta_{2} \pi}{d t}
$$$$
+2 \sqrt{1-e^{2}} \sin ^{2} \frac{i}{2} \frac{d \delta_{2} \delta}{d t} \text {. }
$$ 
Perturbations produced by $\Omega_{3}$ :

$$
\begin{aligned}
& a_{30}=+\frac{3}{20} k_{21} \quad \beta_{30}=+\frac{1}{4} k_{30} a^{\prime} \\
& a_{31}=a_{32}=-\frac{3}{5} \cdot k_{21} \quad \beta_{31}=\beta_{32}=+\frac{3}{8} k_{30} a^{\prime} \\
& a_{33}=+\frac{3}{4} \mathrm{k}_{21}: \quad \beta_{33}=+\frac{15}{4} \mathrm{k}_{30} a^{\prime} \\
& a_{34}=+3 \mathrm{k}_{21} \quad \beta_{34}=+15 \mathrm{k}_{30} \alpha^{\prime} \\
& \beta_{35}=\beta_{36}=+\frac{5}{8} \mathbf{k}_{30} a^{\prime} \\
& c_{300}=-\frac{3}{2}\left(3-5 \cos ^{2} i\right) \cos i \quad c_{320}=+\frac{15}{2} \sin ^{2} i \cos i \quad s_{320}=0 \\
& c_{301}=+\frac{3}{2}\left(1-5 \cos ^{2} i\right) \sin i \quad c_{321}=-\frac{5}{2}\left(1-3 \cos ^{2} i\right) \sin i \quad s_{321}=-\frac{5}{2} \sin 2 i \\
& c_{302}=+\frac{3}{2} \sin ^{2} i \cos i \quad c_{322}=-\frac{1}{2}\left(1-3 \cos ^{2} i\right) \cos i \quad s_{322}=+\cos 2 i \\
& c_{303}=-\frac{3}{2} \sin ^{2} i \\
& c_{323}=-\frac{3}{2}\left(1+\cos ^{2} i\right) \sin i \quad s_{323}=+\frac{3}{2} \sin 2 i \\
& c_{310}=0 \\
& s_{310}=-\frac{3}{4}\left(1-5 \cos ^{2} i\right) \text { sin i } \\
& c_{311}=-\frac{1}{4}\left(1-5 \cos ^{2} i\right) \\
& s_{311}=-\frac{1}{4}\left(11-15 \cos ^{2} i\right) \cos i \\
& c_{312}=+\frac{1}{4} \sin 2 i \\
& s_{312}=+\frac{1}{4}\left(1-3 \cos ^{2} i\right) \text { sin } i \\
& c_{313}=+\frac{3}{4} \sin ^{2} i \\
& s_{313}=+\frac{3}{4} \sin ^{2} i \cos i
\end{aligned}
$$

39 


$$
\begin{aligned}
& \mathrm{F}_{0}^{\prime}=+\alpha_{30} \mathrm{D}_{0}^{\prime}+\beta_{30} \mathrm{E}_{0}^{\prime} \quad \mathrm{K}_{30}^{(\mathrm{c})}=0 \\
& F_{1}^{\prime}=+a_{31} D_{3}^{\prime}+\beta_{31} E_{1}^{\prime} \quad K_{31}^{(c)}=+F_{1}^{\prime} \cos \delta+F_{2}^{\prime} \text { sin } \delta \\
& \mathrm{F}_{2}^{\prime}=+\alpha_{32} \mathrm{D}_{4}^{\prime}+\beta_{32} \mathrm{E}_{2}^{\prime} \quad \mathrm{K}_{32}^{(\mathrm{c})}=-\mathrm{F}_{3}^{\prime} \sin 2 \delta+\frac{1}{2} \mathrm{~F}_{4}^{\prime} \cos 2 \delta \\
& F_{3}^{\prime}=+a_{33} D_{1}^{\prime}+\beta_{33} E_{3}^{\prime} \quad K_{33}^{(c)}=+F_{5}^{\prime} \cos 3 \delta+F_{6}^{\prime} \sin 3 \delta \\
& F_{4}^{\prime}=+\alpha_{34} D_{2}^{\prime}+\beta_{34} E_{4}^{\prime} \quad K_{30}^{(s)}=+F_{0}^{\prime} \\
& \mathrm{F}_{5}^{\prime}=\quad+\beta_{35} \mathrm{E}_{5}^{\prime} \quad \mathrm{K}_{31}^{(\mathrm{s})}=-\mathrm{F}_{1}^{\prime} \sin \delta+\mathrm{F}_{2}^{\prime} \cos \delta \\
& \mathrm{F}_{6}^{\prime}=\quad+\beta_{36} \mathrm{E}_{6}^{\prime} \quad \mathrm{K}_{32}^{(\mathrm{s})}=+\mathrm{F}_{3}^{\prime} \cos 2 \delta+\frac{1}{2} \mathrm{~F}_{4}^{\prime} \sin 2 \delta \\
& \mathrm{K}_{33}^{(s)}=-\mathrm{F}_{5}^{\prime} \sin 3 \delta+\mathrm{F}_{6}^{\prime} \cos 3 \delta \\
& \mathrm{V}_{30}=+c_{300} \mathrm{~K}_{30}^{(\mathrm{s})}+\mathrm{c}_{301} \mathrm{~K}_{31}^{(\mathrm{s})}+\mathrm{c}_{302} \mathrm{~K}_{32}^{(\mathrm{s})}+\mathrm{c}_{303} \mathrm{~K}_{33}^{(\mathrm{s})} \\
& \mathrm{V}_{32}^{(\mathrm{c})}=+\mathrm{c}_{320} \mathrm{~K}_{30}^{(\mathrm{s})}+\mathrm{c}_{321} \mathrm{~K}_{31}^{(\mathrm{s})}+\mathrm{c}_{322} \mathrm{~K}_{32}^{(\mathrm{s})}+\mathrm{c}_{323} \mathrm{~K}_{33}^{(\mathrm{s})} \\
& \mathrm{V}_{32}^{(s)}=\quad+s_{321} K_{31}^{(c)}+s_{322} K_{32}^{(c)}+s_{323} K_{33}^{(c)} \\
& \mathrm{V}_{31}^{(\mathrm{c})}=\quad+\mathrm{c}_{311} \mathrm{~K}_{31}^{(\mathrm{c})}+\mathrm{c}_{312} \mathrm{~K}_{32}^{(\mathrm{c})}+\mathrm{c}_{313} \mathrm{~K}_{33}^{(\mathrm{c})} \\
& V_{31}^{(s)}=s_{310} K_{30}^{(s)}+s_{311} K_{31}^{(s)}+s_{312} K_{32}^{(s)}+s_{313} K_{33}^{(s)}
\end{aligned}
$$

$$
\begin{aligned}
\sin i \frac{d \delta_{3} \delta}{d t} & =+\frac{n a^{3} a^{\prime 3} e}{\left(1-e^{2}\right)^{3}} \cdot \frac{m^{\prime}}{M} \cdot\left(\frac{a^{\prime}}{r^{\prime}}\right)^{3}\left(v_{30} \sin \omega-\frac{1}{2} V_{32}^{(c)} \sin \omega+\frac{1}{2} v_{32}^{(s)} \cos \omega\right) \\
\frac{d \delta_{3} i}{d t} & =+\frac{n a^{3} a^{\prime 3} e}{\left(1-e^{2}\right)^{3}} \cdot \frac{m^{\prime}}{M} \cdot\left(\frac{a^{\prime}}{r^{\prime}}\right)^{3}\left(v_{30} \cos \omega+\frac{1}{2} V_{32}^{(c)} \cos \omega+\frac{1}{2} V_{32}^{(s)} \sin \omega\right)
\end{aligned}
$$




$$
\begin{aligned}
\frac{d \delta_{3} \pi}{d t}= & +\frac{n a^{3} a^{\prime 3}}{\left(1-e^{2}\right)^{3} e} \cdot \frac{m^{\prime}}{M} \cdot\left(\frac{a^{\prime}}{r^{\prime}}\right)^{3}\left(1+4 e^{2}\right)\left(V_{31}^{(c)} \cos \omega+V_{31}^{(s)} \sin \omega\right) \\
& +2 \sin ^{2} \frac{i}{2} \frac{d \delta_{3} \delta}{d t}
\end{aligned}
$$$$
\frac{d \delta_{3} e}{d t}=+\frac{n a^{3} a^{\prime 3}}{\left(1-e^{2}\right)^{2}} \cdot \frac{m^{\prime}}{M} \cdot\left(\frac{a^{\prime}}{r^{\prime}}\right)^{3}\left(V_{31}^{(c)} \sin \omega-V_{31}^{(s)} \cos \omega\right)
$$$$
\frac{d \delta_{3} L}{d t}=+\frac{8 n a^{3} a^{\prime 3} e}{\left(1-e^{2}\right)^{5 / 2}} \cdot \frac{m^{\prime}}{M} \cdot\left(\frac{a^{\prime}}{r^{\prime}}\right)^{3}\left(V_{31}^{(c)} \cos \omega+V_{31}^{(s)} \sin \omega\right)
$$$$
+\frac{\mathrm{e}}{1+\sqrt{1-\mathrm{e}^{2}}} \cdot \frac{\mathrm{d} \delta_{3} \pi}{\mathrm{dt}}+2 \sqrt{1-\mathrm{e}^{2}} \sin ^{2} \frac{\mathrm{i}}{2} \frac{\mathrm{d} \delta_{3} \delta}{\mathrm{dt}}
$$

Perturbations produced by $\Omega_{4}$ :

$$
\begin{array}{ll}
a_{40}=+\frac{3}{56}\left(\mathrm{k}_{20}+\frac{2}{7} \mathrm{k}_{22}\right) \epsilon^{2}-\frac{9}{280} \mathrm{k}_{22} & \beta_{40}=-\frac{1}{28} \mathrm{k}_{31} a^{\prime} \\
a_{41}=a_{42}=+\frac{45}{14}\left(\mathrm{k}_{20}+\frac{1}{7} \mathrm{k}_{22}\right) \epsilon^{2}-\frac{27}{14} \mathrm{k}_{22} & \beta_{41}=\beta_{42}=+\frac{45}{56} \mathrm{k}_{31} \alpha^{\prime}, \\
a_{43}=+\frac{15}{56}\left(\mathrm{k}_{20}-\frac{2}{7} \mathrm{k}_{22}\right) \epsilon^{2}-\frac{9}{16} \mathrm{k}_{22} & \beta_{43}=-\frac{15}{28} \mathrm{k}_{31} \alpha^{\prime} \\
a_{44}=+\frac{15}{14}\left(\mathrm{k}_{20}-\frac{2}{7} \mathrm{k}_{22}\right) \epsilon^{2}-\frac{9}{4} \mathrm{k}_{22} & \beta_{44}=-\frac{15}{7} \mathrm{k}_{31} \alpha^{\prime} . \\
a_{45}=a_{46}=0 & \beta_{45}=\beta_{46}=+\frac{5}{8} \mathrm{k}_{31} \alpha^{\prime}
\end{array}
$$




$$
\begin{aligned}
& c_{400}=+\frac{3}{8}\left(3-30 \cos ^{2} i+35 \cos ^{4} i\right) \\
& c_{401}=+\frac{1}{16}\left(3-7 \cos ^{2} i\right) \sin 2 i \\
& c_{402}=-\frac{3}{8}\left(1-7 \cos ^{2} i\right) \sin ^{2} i \\
& c_{403}=-\frac{3}{8} \sin ^{3} i \cos i \\
& c_{420}=-\frac{5}{2}\left(1-7 \cos ^{2} i\right) \sin ^{2} i \quad s_{420}=0 \\
& c_{421}=-\frac{1}{12}\left(4-7 \cos ^{2} i\right) \sin 2 i \quad s_{421}=-\frac{1}{12}\left(1-7 \cos ^{2} i\right) \sin i \\
& c_{422}=+\frac{1}{2}\left(1-6 \cos ^{2} i+7 \cos ^{4} i\right) \quad s_{422}=+\frac{1}{2}\left(5-7 \cos ^{2} i\right) \cos i \\
& \mathrm{c}_{423}=-\frac{1}{2} \cos ^{3} \mathrm{i} \sin \mathrm{i} \quad \mathrm{s}_{423}=+\frac{1}{4}\left(1-3 \cos ^{2} \mathrm{i}\right) \sin \mathrm{i} \\
& c_{410}=0 \\
& s_{410}=+\frac{15}{2}\left(3-7 \cos ^{2} i\right) \sin 2 i \\
& c_{411}=-\frac{1}{4}\left(3-7 \cos ^{2} i\right) \cos i \\
& s_{411}=-\frac{1}{4}\left(3-27 \cos ^{2} i+28 \cos ^{4} i\right) \\
& c_{412}=-\frac{3}{2}\left(1-7 \cos ^{2} i\right) \sin i \\
& s_{412}=-\frac{3}{2}\left(4-7 \cos ^{2} i\right) \sin 2 i \\
& c_{413}=+\frac{9}{4} \sin ^{2} i \cos i \\
& s_{413}=+\frac{3}{4}\left(1-4 \cos ^{2} i\right) \sin ^{2} i
\end{aligned}
$$




$$
\begin{aligned}
& c_{430}=0 \\
& s_{430}=-35 \sin ^{3} i \cos i \\
& c_{431}=+\frac{7}{4} \sin ^{2} i \cos i \\
& s_{431}=+\frac{7}{12} \sin ^{2} \cdot i\left(1-4 \cos ^{2} i\right) \\
& c_{432}=+\frac{7}{2}\left(1-3 \cos ^{2} i\right) \sin i \quad s_{432}=-7 \sin i \cos ^{3} i \\
& c_{433}=-\frac{1}{4}\left(5-9 \cos ^{2} i\right) \cos i \quad s_{433}=+\frac{1}{4},\left(3-3 \cos ^{2} i-4 \cos ^{4} i\right) \\
& G_{0}^{\prime}=\alpha_{40} D_{0}^{\prime}+\beta_{40} E_{0}^{\prime} \\
& \mathrm{G}_{1}^{\prime}=\alpha_{41} \mathrm{D}_{3}^{\prime}+\beta_{41} \mathrm{E}_{1}^{\prime} \\
& G_{2}^{\prime}=a_{42} D_{4}^{\prime}+\beta_{42} E_{2}^{\prime} \\
& G_{3}^{\prime}=a_{43} D_{1}^{\prime}+\beta_{43} E_{3}^{\prime} \\
& G_{4}^{\prime}=\alpha_{44} D_{2}^{\prime}+\beta_{44} E_{4}^{\prime} \\
& \mathrm{G}_{5}^{\prime}=\quad \beta_{45} \mathrm{E}_{5}^{\prime} \\
& G_{6}^{\prime}=\quad \beta_{46} E_{6}^{\prime} \\
& \mathrm{K}_{40}^{(c)}=\mathrm{G}_{0}^{\prime} \\
& \mathrm{K}_{41}^{(c)}=+\mathrm{G}_{1}^{\prime} \sin \delta-\mathrm{G}_{2}^{\prime} \cos \delta \\
& \mathrm{K}_{41}^{(s)}=+\mathrm{G}_{1}^{\prime} \cos \delta \delta+\mathrm{G}_{2}^{\prime} \sin \delta \\
& \mathrm{K}_{42}^{(\mathrm{c})}=+\mathrm{G}_{3}^{\prime} \cos 2 \delta_{0}+\frac{1}{2} \mathrm{G}_{4}^{\prime} \text { s in } 2 \delta_{0} \\
& \mathrm{~K}_{42}^{(\mathrm{s})}=+\mathrm{G}_{3}^{\prime} \sin 2 \delta-\frac{1}{2} \mathrm{G}_{4}^{\prime} \cos 2 \delta
\end{aligned}
$$




$$
\begin{aligned}
& \mathrm{K}_{43}^{(c)}=+\mathrm{G}_{5}^{\prime} \sin 3 \delta-\mathrm{G}_{6}^{\prime} \cos 3 \dot{\delta} \\
& \mathrm{K}_{43}^{(s)}=+\mathrm{G}_{5}^{\prime} \cos 3 \delta+\mathrm{G}_{6}^{\prime} \sin 3 \Omega \\
& V_{40}=+c_{400} K_{40}^{(c)}+c_{401} K_{41}^{(c)}+c_{402} K_{42}^{(c)}+c_{403} K_{43}^{(c)} \\
& \mathrm{V}_{42}^{(c)}=+c_{420} \mathrm{~K}_{40}^{(c)}+c_{421} \mathrm{~K}_{41}^{(\mathrm{c})}+\mathrm{c}_{422} \mathrm{~K}_{42}^{(\mathrm{c})}+\mathrm{c}_{423} \mathrm{~K}_{43}^{(\mathrm{c})} \\
& V_{42}^{(s)}=\quad+s_{421} K_{41}^{(s)}+s_{422} K_{42}^{(s)}+s_{423} K_{43}^{(s)} \\
& \mathrm{V}_{41}^{(c)}=\quad+\mathrm{c}_{411} \mathrm{~K}_{41}^{(\mathrm{s})}+\mathrm{c}_{412} \mathrm{~K}_{42}^{(\mathrm{s})}+\mathrm{c}_{413} \mathrm{~K}_{43}^{(\mathrm{s})} \\
& \mathrm{V}_{41}^{(s)}=+\mathrm{s}_{410} \mathrm{~K}_{40}^{(c)}+\mathrm{s}_{411} \mathrm{~K}_{41}^{(\mathrm{c})}+\mathrm{s}_{412} \mathrm{~K}_{42}^{(c)}+\mathrm{s}_{413} \mathrm{~K}_{43}^{(\mathrm{c})} \\
& \mathrm{V}_{43}^{(s)}=\quad+\mathrm{c}_{431} \mathrm{~K}_{41}^{(\mathrm{s})}+\mathrm{c}_{432} \mathrm{~K}_{42}^{(\mathrm{s})}+\mathrm{c}_{433} \mathrm{~K}_{43}^{(\mathrm{s})} \\
& V_{43}^{(s)}=+s_{430} K_{40}^{(c)}+s_{431} K_{41}^{(c)}+s_{432} K_{42}^{(c)}+s_{433} K_{43}^{(c)}
\end{aligned}
$$

$\sin i \frac{d \delta_{4} \delta}{d t}=+\frac{n a^{4} \alpha^{\prime 3}}{2\left(1-e^{2}\right)^{4}} \cdot \frac{m^{\prime}}{M} \cdot\left(\frac{a^{\prime}}{r^{\prime}}\right)^{3} \cdot\left\{+\left(1+\frac{3}{2} e^{2}\right) V_{41}^{(s)}\right.$

$$
\left.+\frac{3}{4} e^{2}\left[+\left(-V_{41}^{(s)}+V_{43}^{(s)}\right) \cos 2 \omega+\left(+V_{41}^{(c)}-V_{43}^{(c)}\right) \sin 2 \omega\right]\right\}
$$

$$
\begin{aligned}
\frac{d \delta_{4} i}{d t}= & +\frac{n a^{4} \alpha^{\prime 3}}{2\left(1-e^{2}\right)^{4}} \cdot \frac{m^{\prime}}{M} \cdot\left(\frac{a^{\prime}}{r^{\prime}}\right)^{3} \cdot\left\{\left(1+\frac{3}{2} e^{2}\right) V_{41}^{(c)}\right. \\
& \left.+\frac{3}{4} e^{2}\left[+\left(+V_{41}^{(c)}+V_{43}^{(c)}\right) \cos 2 \omega+\left(+V_{41}^{(s)}+V_{43}^{(s)}\right) \sin 2 \omega\right]\right\}
\end{aligned}
$$

$$
\begin{aligned}
\frac{\mathrm{d} \delta_{4} \pi}{\mathrm{dt}}= & +\frac{\mathrm{n} \alpha^{4} \alpha^{\prime 3}}{\left(1-\mathrm{e}^{2}\right)^{4}} \cdot \frac{\mathrm{m}^{\prime}}{\mathrm{M}} \cdot\left(\frac{\mathrm{a}^{\prime}}{\mathrm{r}^{\prime}}\right)^{3} \cdot\left\{+\left(+10+\frac{15}{2} \mathrm{e}^{2}\right) \mathrm{V}_{40}\right. \\
& \left.+\left(\frac{3}{2}+\frac{15}{4} \mathrm{e}^{2}\right)\left(+\mathrm{V}_{42}^{(\mathrm{c})} \cos 2 \omega+\mathrm{V}_{42}^{(\mathrm{s})} \sin 2 \omega\right)\right\}+2 \sin ^{2} \frac{\mathrm{i}}{2} \frac{\mathrm{d} \delta_{4} \delta}{\mathrm{dt}}
\end{aligned}
$$




$$
\begin{aligned}
\frac{\mathrm{d} \delta_{4} \mathrm{e}}{\mathrm{dt}}= & +\frac{3 \mathrm{n} a^{4} a^{\prime 3}}{2\left(1-\mathrm{e}^{2}\right)^{3}} \cdot \frac{\mathrm{m}^{\prime}}{\mathrm{M}} \cdot\left(\frac{\mathrm{a}^{\prime}}{\mathrm{r}^{\prime}}\right)^{3} \cdot\left(\mathrm{V}_{42}^{(\mathrm{c})} \sin 2 \omega-\mathrm{V}_{42}^{(\mathrm{s})} \cos 2 \omega\right) \\
\frac{\mathrm{d} \delta_{4} \mathrm{~L}}{\mathrm{dt}}= & +\frac{10 \mathrm{n} a^{4} \alpha^{\prime 3}}{\left(1-\mathrm{e}^{2}\right)^{7 / 2}} \cdot \frac{\mathrm{m}^{\prime}}{\mathrm{M}} \cdot\left(\frac{\mathrm{a}^{\prime}}{\mathrm{r}^{\prime}}\right)^{3} \cdot\left\{\left(1+\frac{3}{2} \mathrm{e}^{2}\right) \mathrm{V}_{40}+\frac{3}{4} \mathrm{e}^{2}\left(\mathrm{~V}_{42}^{(\mathrm{c})} \cos 2 \omega+\mathrm{V}_{42}^{(\mathrm{s})} \sin 2 \omega\right)\right\} \\
& +\frac{\mathrm{e}}{1+\sqrt{1-\mathrm{e}^{2}}} \cdot \frac{\mathrm{d} \delta_{4} \pi}{\mathrm{dt}}+2 \sqrt{1-\mathrm{e}^{2}} \sin ^{2} \frac{\mathrm{i}}{2} \frac{\mathrm{d} \delta_{4} \delta}{\mathrm{dt}}
\end{aligned}
$$

Indirect effects (Kozai, 1965):

$$
\begin{aligned}
& \frac{d \delta_{5} \delta}{d t}=-\left(n_{0} \delta_{1} \tan i\right) \delta i \\
& \frac{d \delta_{5} \pi}{d t}=(+5 \sin i-\tan i) n_{0} \delta_{1} \delta i \\
& \frac{d \delta_{5} L}{d t}=(+8 \sin i-\tan i) n_{0} \delta_{1} \delta i
\end{aligned}
$$




\section{REFERENCES}

Kozai, Y., "Effects of the Tidal Deformation of the Earth on the Motion of Close Earth Satellites," Publ. Astron. Soc. Japan, 17, p. 395, 1965.

Musen, P. and Estes, R., "On the Tidal Effects in the Motion of Artificial Satellites," NASA-X-550-71-342, August 1971. Celestial Mechanics ( in press).

Newton, R. R., "A Satellite Determination of Tidal Parameters and Earth Deceleration." Geoph. J. R. Astr. Soc. 14, pp. 505-539, 1968.

Smith, D. E., Kolenkiewicz, R. and Dunn, P. J., "Geodetic Studies by Laser Ranging to Satellites," NASA-X-553-71-360, April 1971. 